University of Nebraska - Lincoln

DigitalCommons@University of Nebraska - Lincoln

July 2007

\title{
Epitaxial growth and surface properties of half-metal NiMnSb films
}

C. N. Borca

Swiss Light Source and the Laboratory for Waste Management, Paul Scherrer Institute, Viligen PSI

$\mathrm{CH}-5232$, Switzerland

D. Ristoiu

Laboratoire Louis Néel, CNRS, BP166X, 38042 Grenoble Cedex, France

H.-K. Jeong

SungkyunKwan University, hjeong@unl.edu

Takashi Komesu

University of Nebraska-Lincoln, tkomesu2@unl.edu

A. N. Caruso

North Dakota State University, carusoan@umkc.edu

See next page for additional authors

Follow this and additional works at: https://digitalcommons.unl.edu/physicsdowben

Part of the Physics Commons

Borca, C. N.; Ristoiu, D.; Jeong, H.-K.; Komesu, Takashi; Caruso, A. N.; Pierre, J.; Ranno, L.; Nozieres, J. P.; and Dowben, Peter A., "Epitaxial growth and surface properties of half-metal NiMnSb films" (2007). Peter Dowben Publications. 187.

https://digitalcommons.unl.edu/physicsdowben/187

This Article is brought to you for free and open access by the Research Papers in Physics and Astronomy at DigitalCommons@University of Nebraska - Lincoln. It has been accepted for inclusion in Peter Dowben Publications by an authorized administrator of DigitalCommons@University of Nebraska - Lincoln. 


\section{Authors}

C. N. Borca, D. Ristoiu, H.-K. Jeong, Takashi Komesu, A. N. Caruso, J. Pierre, L. Ranno, J. P. Nozieres, and Peter A. Dowben 


\title{
Epitaxial growth and surface properties of half-metal NiMnSb films
}

\author{
C. N. Borca ${ }^{1}$, D. Ristoiu ${ }^{2}$, H-K. Jeong ${ }^{3}$, Takashi Komesu ${ }^{4}$,
} A. N. Caruso ${ }^{5}$, J. Pierre ${ }^{2}$, L. Ranno ${ }^{2}$, J. P. Nozières ${ }^{2}$, and P. A. Dowben ${ }^{4}$

${ }^{1}$ Swiss Light Source and the Laboratory for Waste Management, Paul Scherrer Institute, Viligen PSI CH-5232, Switzerland

${ }^{2}$ Laboratoire Louis Néel, CNRS, BP166X, 38042 Grenoble Cedex, France

${ }^{3}$ Department of Physics, SungkyunKwan University, Jangan Suwon 440-746, Korea

${ }^{4}$ Department of Physics and Astronomy and the Center for Materials Research and Analysis, Behlen Laboratory of Physics, University of Nebraska-Lincoln, Lincoln, NE 68588-0111, USA

${ }^{5}$ Center for Nanoscale Science and Engineering, North Dakota State University, Fargo, ND 58047, USA

Corresponding author: C. N. Borca, email: camelia.borca@psi.ch

\begin{abstract}
We present, herein, an extended study of the half-Heusler alloy NiMnSb, starting with the deposition technique, continuing with the basic structural and magnetic properties of the thin films, and finishing with the electronic and compositional properties of their surfaces. The experimental methods we apply combine magnetization and magnetoresistivity measurements, atomic force microscopy, ferromagnetic resonance, $x$-ray and neutron diffraction, low energy electron diffraction, angle resolved x-ray photoemission, extended x-ray absorption fine structure spectroscopy, soft x-ray magnetic circular dichroism and spin polarized inverse photoemission spectroscopy. We find that stoichiometric surfaces exhibit close to $100 \%$ spin polarization at the centre of the surface Brillouin zone at the Fermi edge at ambient temperatures. There is strong evidence for a moment reordering transition at around $80 \mathrm{~K}$ which marks the crossover from a high polarization state $(T<80 \mathrm{~K})$ to a more representative metallic ferromagnetic state $(T>80 \mathrm{~K})$. The results from the different experimental techniques are successively reviewed, with special emphasis on the interplay between composition and electronic structure of the NiMnSb film surfaces. Surface segregation, consistent with a difference in free enthalpy between the surface and the bulk, is induced by annealing treatments. This surface segregation greatly reduces the surface polarization.
\end{abstract}




\section{Introduction}

Half-metals, first postulated by de Groot in the early 1970s, can be considered as hybrids between metals and semiconductors, since the majority spin band structure shows a metallic behavior, while the minority spin band structure exhibits a gap at the Fermi level such as in a semiconductor. Due to the gap for one spin direction, the density of states at the Fermi level has, theoretically, 100\% spin polarization. Such fully spin polarized ferromagnetic materials are of considerable interest in the newly developed area of spin electronics (or spintronics). Among the most cited candidates for $100 \%$ spin polarization are the semiHeusler alloys (NiMnSb) [1-9], full Heusler alloys [10, 11], zinc-blended structure materials [12-14], colossal magnetoresistance materials $\left(\mathrm{La}_{1-x} \mathrm{Sr}_{x} \mathrm{MnO}_{3}[15,16], \mathrm{Sr}_{2} \mathrm{FeMoO}_{6}\right.$ [17]), and semimetallic magnetic oxides $\left(\mathrm{CrO}_{2}[18-21]\right.$ and $\mathrm{Fe}_{3} \mathrm{O}_{4}$ [22, 23]). A number of other materials have also been suggested as half-metallic ferromagnets [24, 25].

$\mathrm{NiMnSb}$ was the first ferromagnet where half-metallic character at low temperature was predicted from band structure calculations [1]. Support for the half-metallic character of NiMnSb was significantly strengthened by the experimental studies of Hanssen et al. [5], by comparing the angular correlations of positron annihilation with theoretical calculations. Numerous experimental efforts have, however, failed to prove the existence of full $100 \%$ polarization of conduction electrons at the Fermi level. All experiments performed to test the electronic properties near the surface lead to polarizations between 40 and $58 \%$ [26-31]. Nearly $100 \%$ polarization was reported on $\mathrm{NiMnSb}(100)$ sputtered thin films using spin polarized inverse photoemission [32-34]. However, these measurements were $k$ resolved and therefore do not directly demonstrate the half-metallic character across the entire Brillouin zone. No claim was made that these measurements did indeed prove halfmetallic character [32-34].

Several explanations have been put forward to account for the lower than expected polarization measured in [26-31]. First, it is known that at finite temperature, there exists a non-zero density of states in the minority channel due to magnons leading to a polarization lower than 100\% [35-41]. Indeed, at any finite temperature, there will be electron-phonon and/or magnon-phonon coupling [36-38], or state rehybridization [41] so that polarization could decrease dramatically well below the Curie temperature. Other possibilities for significantly lower measured polarization could be related to the presence of spin minority surface states $[9,38,42-46]$, surface segregation $[32,47,48]$ or a variety of defects $[31$, $49,50]$ which make the surface a different material from the bulk. Finally, the NiMnSb may not be a true half-metallic ferromagnet above $80 \mathrm{~K}[33,41,51,52]$ though this would not explain the measured low polarization and magnetoresistance well below $80 \mathrm{~K}$.

This paper presents experimental evidence that suggest that there are significant changes in electronic properties near a metamagnetic transition temperature of $80 \mathrm{~K}$, far below the Curie point of $\mathrm{NiMnSb}\left(T_{\mathrm{C}}=730 \mathrm{~K}\right)$. At a temperature of about $80 \mathrm{~K}$, there is a crossover from the high polarization metallic phase at lower temperatures to a more normal ferromagnet at higher temperatures is associated with a change in the magnetic moment and hybridization as a function of temperature. This summary of our experimental work, made on this system over the last six years, is now increasingly consistent with theoretical expectations [35-38, 41]. The structural, magnetic and electronic properties of the thin films, as well as their surfaces, suggests that a compelling experimental "proof" of the half-metallic character remains elusive.

\section{Epitaxy and morphology of the NiMnSb films on MgO substrates}

$\mathrm{NiMnSb}$ (100) films have been deposited using a DC facing targets sputtering (FTS) system with three pairs of FTS (facing target sputtering) electrodes and one planar magne- 


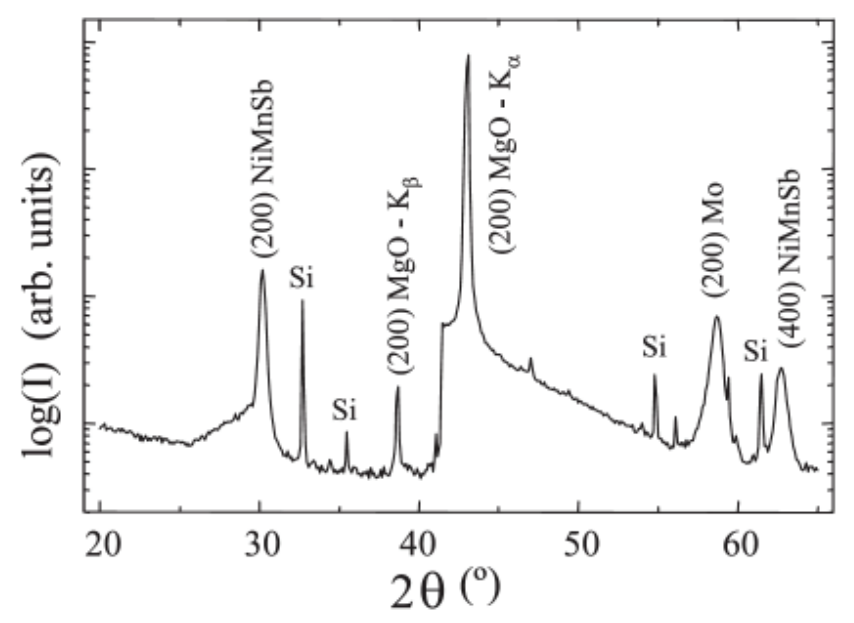

Figure 1. $\theta-2 \theta$ diffraction diagram for an NiMnSb $500 \AA / \mathrm{Mo} / \mathrm{MgO}$ film deposited at $400{ }^{\circ} \mathrm{C}$ with $P_{\mathrm{Ar}}=10 \mathrm{mTorr}$. The non-indexed peaks correspond to the Si sample holder. Adapted from [53].

tron. More details about the deposition process can be found in [53]. The main reason for exploiting FTS lies in the stoichiometric deposition which enables the use of a single 1:1:1 target. The chemically cleaned $\mathrm{MgO}$ monocrystalline (100) substrates were first baked for $2 \mathrm{~h}$ at $500{ }^{\circ} \mathrm{C}$ in order to remove the surface hydroxide layer and then set at the deposition temperature. A Mo seed layer was then sputtered with the planar magnetron at $P_{\mathrm{Ar}}=10 \mathrm{mTorr}$ with a deposition rate of $0.7 \mathrm{As}^{-1}$ and a deposition temperature between 250 and $550{ }^{\circ} \mathrm{C}$. Immediately after the deposition of the seed layer, the NiMnSb layer was sputtered at the same temperature and argon pressure, with a deposition rate of the order of $1 \AA \mathrm{s}^{-1}$. A $150 \AA \mathrm{Sb}$ capping layer was finally deposited in order to prevent the any oxidation of the NiMnSb layer prior to introduction into ultrahigh vacuum for the surface science and electron spectroscopy studies. Deposition temperatures higher than $250{ }^{\circ} \mathrm{C}$ were used as $\mathrm{NiMnSb}$ growth on Si substrates exhibited poor crystallization for temperatures lower than $275{ }^{\circ} \mathrm{C}$ [54]. Rutherford backscattering (RBS) analysis indicated a 1:1:1 stoichiometry in all samples.

The crystalline structure and extent of crystallinity of the films were confirmed by means of $\theta-2 \theta$ of x-ray diffraction (XRD) as well as by grazing x-ray diffraction (GXRD), both using $\mathrm{Cu} \mathrm{K \alpha}$ radiation. The XRD results in figure 1 , for the range $20^{\circ}<2 \theta<65^{\circ}$ from a film deposited at $400{ }^{\circ} \mathrm{C}$, show only the (200) Mo peak and the (200) and (400) $\mathrm{NiMnSb}$ peaks. This indicates a strong (100) growth texture for both Mo and the NiMnSb. The same $\theta-2 \theta$ diagram was obtained for samples grown throughout the temperature range $350-650{ }^{\circ} \mathrm{C}$. For deposition at temperatures higher than $550{ }^{\circ} \mathrm{C}$, RBS analysis indicates that interdiffusion occurs at the $\mathrm{Mo} / \mathrm{NiMnSb}$ interface. Complementary grazing $\mathrm{X}$ ray diffraction (GXRD) experiments were performed to differentiate between simply textured films and real epitaxial growth. A GXRD diagram obtained on a $500 \AA$ NiMnSb film deposited at $400{ }^{\circ} \mathrm{C}$ is presented in figure 2 .

Clear evidence of epitaxy was found from GXRD, with epitaxial relationships of $(100) \mathrm{NiMnSb} /(100) \mathrm{Mo} /(110) \mathrm{MgO}$. Such relative orientations are explained by considering the lattice parameters of $\mathrm{MgO}\left(a_{\mathrm{MgO}}=4.213 \AA\right)$ and $\mathrm{NiMnSb}\left(a_{\mathrm{NiMnSb}}=5.905 \AA\right)$, both presenting an fcc structure, and of Mo $\left(a_{\mathrm{Mo}}=3.147 \AA\right.$ ) presenting a bcc structure. As $\sqrt{2} a_{\mathrm{MgO}} \approx 2 a_{\mathrm{Mo}}$ (with a misfit 5.2\%), a rotation by $45^{\circ}$ tilt is expected for Mo on $\mathrm{MgO}$, whereas as $a_{\mathrm{NiMnSb}} \approx 2 . a_{\mathrm{Mo}}$ (a misfit of $5.8 \%$ ), so a cube on cube epitaxy is expected for $\mathrm{NiMnSb}$ on Mo. The schematic of the epitaxial relations between NiMnSb, Mo and MgO 


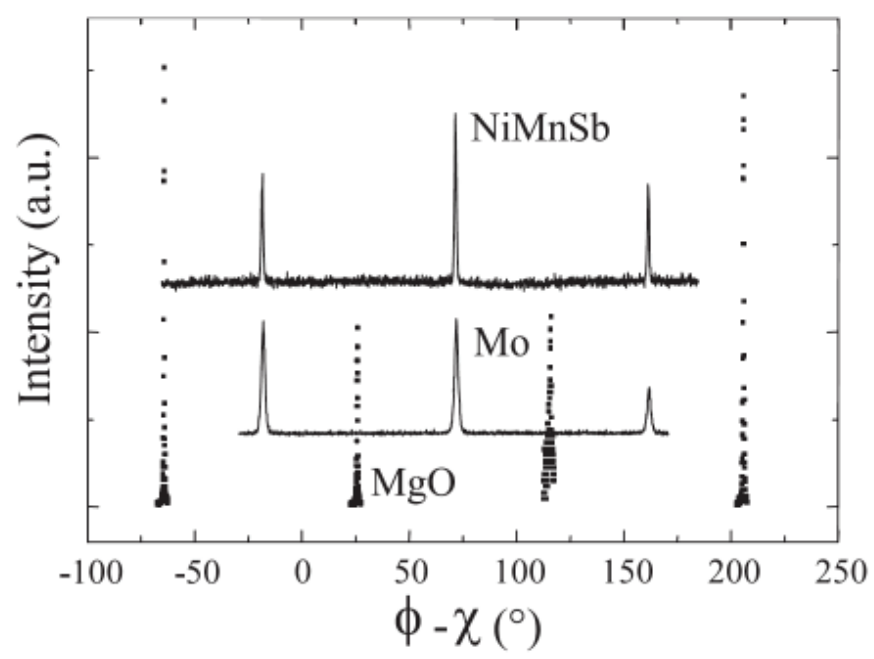

Figure 2. GXRD diagram of a NiMnSb $500 \AA \mathrm{A} / \mathrm{Mo} / \mathrm{MgO}$ film deposited at $400{ }^{\circ} \mathrm{C}$ with $P_{\mathrm{Ar}}=10$ mTorr. Adapted from [53].
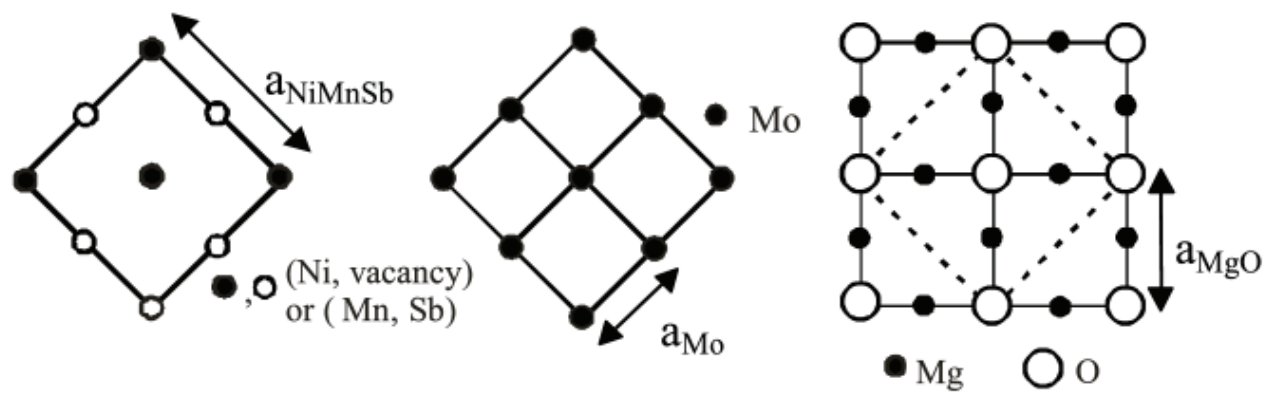

Figure 3. Schematic of the stacked epitaxial correspondences between NiMnSb, Mo and $\mathrm{MgO}$, viewed in the (001) plane.

is shown in figure 3 using the (001) projection. The mosaicity is found to be $1.5^{\circ}$ for Mo and $2.5^{\circ}$ for $\mathrm{NiMnSb}$ which indicates the rather good quality of the epitaxial growth. The Mo mosaicity is temperature independent, but in the case of NiMnSb the mosaicity was found to be larger for lower temperatures. Although $\mathrm{MgO}$ and $\mathrm{NiMnSb}$ present a smaller lattice mismatch $\left(\sqrt{2} \cdot a_{\mathrm{MgO}} \approx a_{\mathrm{NiMnSb}}\right.$, a misfit of $\left.1 \%\right)$, NiMnSb grown directly on $\mathrm{MgO}$ exhibits (200) textured growth but the films were polycrystalline in all the temperature ranges studied. This is most likely due to the wetting (surface energy) of the $\mathrm{MgO}$ substrate, being more favourable for Mo buffer layer than for NiMnSb films.

The NiMnSb(100) surface morphology was investigated by atomic force microscopy (AFM) in contact mode. The AFM image of the Mo seed layer deposited at $400{ }^{\circ} \mathrm{C}$ is shown in figure 4 . The Mo thin film surface is continuous and flat with an average roughness of $5 \AA$ over a $10 \mu \mathrm{m}^{2}$ surface. The AFM image of an epitaxial NiMnSb film grown at $450{ }^{\circ} \mathrm{C}$, under $10 \mathrm{mTorr}$ of argon, is shown in figure 5(a). The film is discontinuous with rather flat islands (rms 5-7 $\AA$ ) of square-like shapes and furrows along the [110] directions as deep as the film thickness. A tendency to percolation was observed with increasing thickness, although total percolation was not achieved in the thickness range studied here. To improve the surface quality and to achieve the necessary two-dimensional 


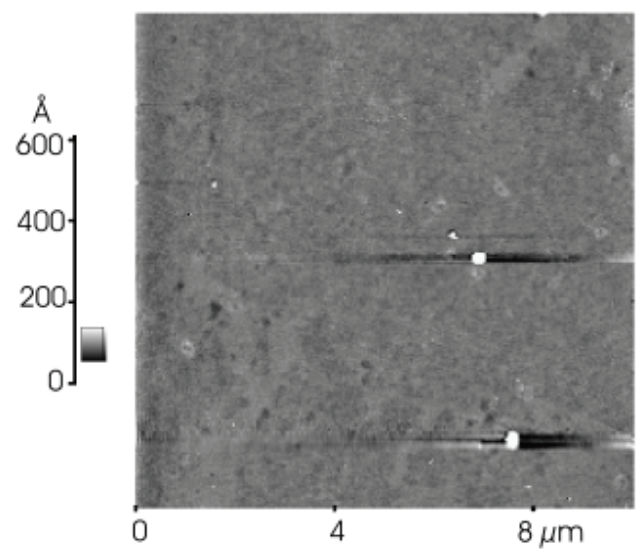

Figure 4. AFM image of a $10 \times 10 \mu \mathrm{m}^{2} \mathrm{Mo} / \mathrm{MgO}$ surface deposited at $400{ }^{\circ} \mathrm{C}$. Adapted from [53].

growth required for transport measurements as well as the surface and polarization studies, we varied the deposition temperature and Ar pressure (figures 5(b) and (c)). The optimum deposition conditions for NiMnSb on Mo appear to be $T=400{ }^{\circ} \mathrm{C}, P_{\mathrm{Ar}}=3 \mathrm{mTorr}$ and a deposition rate of $0.6 \AA \mathrm{s}^{-1}$. The AFM surface image of such a film shows an average roughness of about $8-9 \AA$ (rms), has a mosaic spread of $2.3^{\circ}$ and the film is fully continuous as presented in figure $5(\mathrm{~d})$.

Increasing the growth temperature promotes the three-dimensional growth with better defined square islands. This can be understood by considering nucleation and growth thermodynamics [55]: the higher incident energy leads to a larger mobility of the adatoms and therefore to an island-like growth in the case of positive interface energy (as it appears to be the case here). High argon pressure also favors discontinuous growth (although not as dramatically as temperature) as generally observed for sputtered films [56]. Modest changes in the deposition rate, in the range studied here $\left(0.5-1 \AA \mathrm{s}^{-1}\right)$, had no effect.

In order to ascertain the short range crystalline order in the NiMnSb films, we performed EXAFS (extended x-ray absorption fine structure) spectroscopy. The resulting oscillations of the measured absorption coefficient are characteristic of the chemical and structural environment of the absorbing atom. The short range three-dimensional $\mathrm{NiMnSb}$ thin film structure can be determined by fitting these oscillations. The EXAFS spectra were recorded at the D42 experimental station of the DCI storage ring at LURE, Orsay (France). Measurements were performed in the reflection mode, detecting the total electron yield (TEY) for probing the absorption coefficient above the K edges of $\mathrm{Mn}$ and $\mathrm{Ni}$. The absorption spectra were normalized by the incident photon flux and performed using a $\operatorname{Si}(331)$ channel-cut monochromator.

The EXAFS measurements were performed using two geometries, i.e., with sample oriented parallel or perpendicular to the direction of the x-ray polarization vector, in order to probe the three-dimensional structure of the NiMnSb films. The overlap between the two L edges of $\mathrm{Sb}\left(\mathrm{L}_{\mathrm{I}}\right.$ and $\left.\mathrm{L}_{\mathrm{II}}\right)$ produced "beating nodes" in the EXAFS oscillations, making the data analysis intractable for the $\mathrm{Sb}$ atom shell. Therefore, we limited our measurements to the Ni and Mn K edges. After background subtraction, the EXAFS oscillations were extracted and normalized to the height of the absorption step edge. The measured spectra were analyzed using standard methods [57]. The theoretical EXAFS oscillations (amplitudes and phases) were calculated using McKale's method [58]. The fitting parameters were restricted to the number of atoms in the first coordination shell $j\left(N_{j}\right)$, the distance between those atoms and the probed atom $\left(R_{j}\right)$ and the Debye-Waller factors $\left(\sigma_{j}\right)$. 

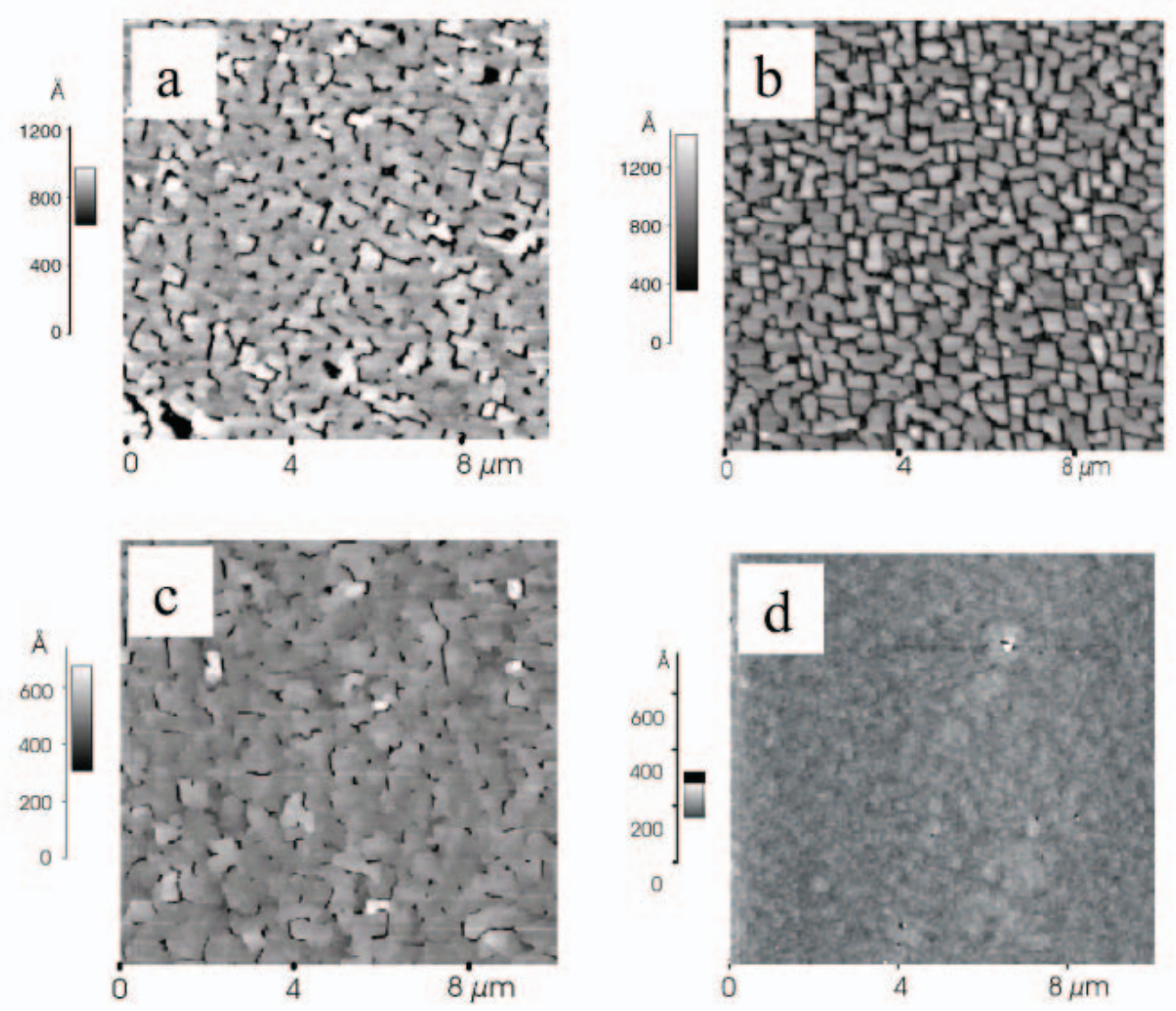

Figure 5. (a)AFM image of a $10 \times 10 \mu \mathrm{m}^{2}$ surface of $\mathrm{NiMnSb} / \mathrm{Mo} / \mathrm{MgO}$ grown at $T=450$ ${ }^{\circ} \mathrm{C}$ and $P_{\mathrm{Ar}}=10 \mathrm{mTorr}$. (b) AFM image for $T=550{ }^{\circ} \mathrm{C}$ and $P_{\mathrm{Ar}}=10 \mathrm{mTorr}$. (c) AFM image for $T=450{ }^{\circ} \mathrm{C}$ and $P_{\mathrm{Ar}}=3 \mathrm{mTorr}$. (d) AFM image for $T=400{ }^{\circ} \mathrm{C}$ and $P_{\mathrm{Ar}}=3 \mathrm{mTorr}$.

In the case of a perfect $\mathrm{NiMnSb}$ structure, a $\mathrm{Mn}$ atom has four $\mathrm{Ni}$ atoms in the first coordination sphere at a distance of $a^{\sqrt{3}} / 4 \cong 2.55 \AA$ and, in a second coordination sphere, six $\mathrm{Sb}$ atoms at a distance of $a / 2 \cong 2.95 \AA$, where $a=5.9 \mathrm{~A}$ is the lattice parameter of NiMnSb. The EXAFS simulations were performed with two coordination spheres about $\mathrm{Mn}$, the first one containing $\mathrm{Ni}$ atoms and the second one $\mathrm{Sb}$ atoms, and with the corresponding phase $\varphi_{j}(k)$ and amplitude $F_{j}(k)$ functions, as indicated by a continuous line in figure 6 for (a) the parallel and (b) the perpendicular configurations. The Ni atom is surrounded at equal distance of $a^{\sqrt{3}} / 4 \cong 2.55 \AA$ by four $\mathrm{Mn}$ atoms and four $\mathrm{Sb}$ atoms. We were forced to use two coordination shells for the simulation of the data from $\mathrm{Ni}$, since the code used does not accept two atomic species situated in the same shell, and the fittings to the data are shown in figure 7 (the continuous lines). For both $\mathrm{Mn}$ and $\mathrm{Ni}$, there is good agreement between experimental and calculated $k$-weighted amplitudes for both (a) the parallel and (b) the perpendicular configurations. For the data from $\mathrm{Mn}$, the calculated Debye-Waller factors are 0.06 for $\mathrm{Ni}$ and 0.065 for $\mathrm{Sb}$, while for the EXAFS data from Ni, the Debye-Waller factors are 0.062 for the $\mathrm{Mn}$ atoms and 0.058 for the $\mathrm{Sb}$ atoms.

The agreement in bond lengths and shell occupation, between theoretical values and those abstracted from the EXAFS data, as summarized in table 1 for the coordination 


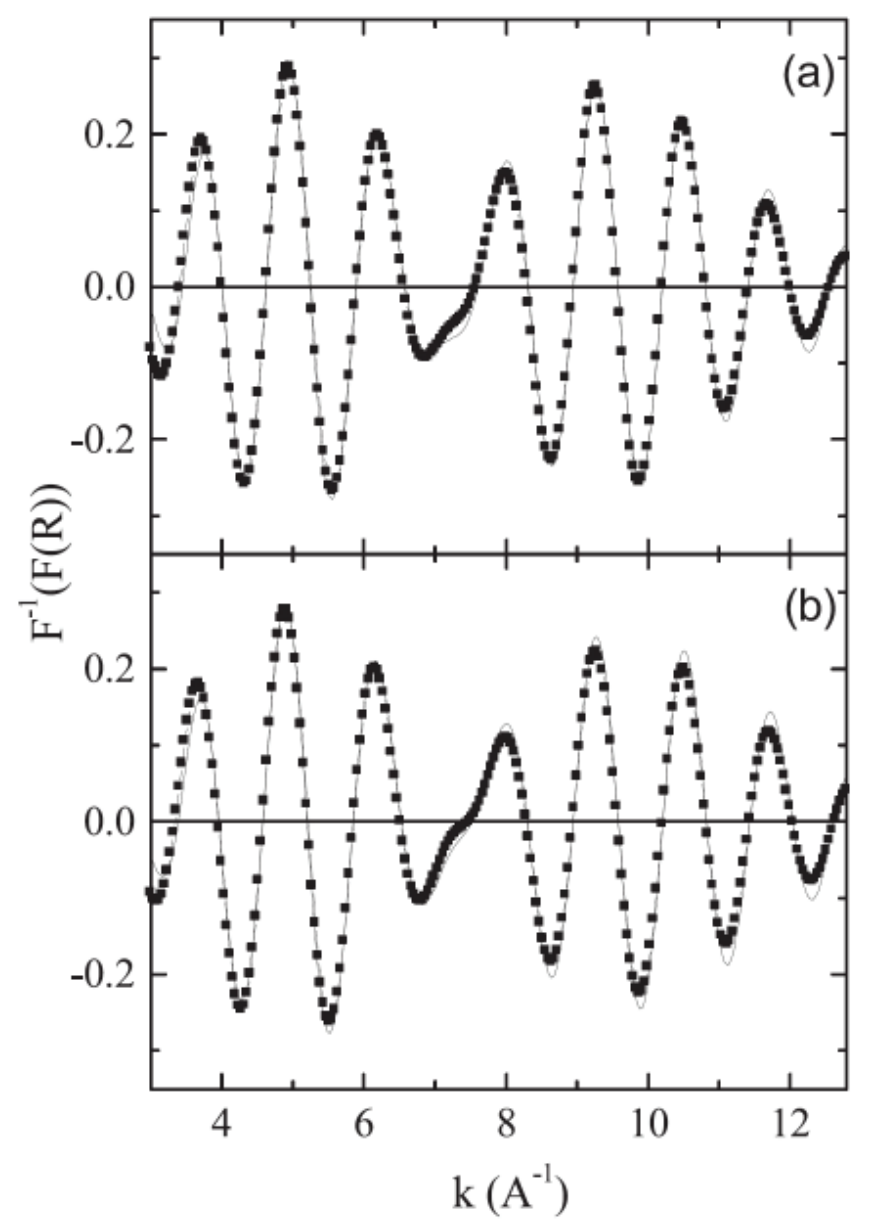

Figure 6. $k$-weighted EXAFS spectra recorded at the Mn edge in parallel (a) and perpendicular (b) configurations. The solid lines correspond to the simulations of the NiMnSb structure while the dots represent the experimental measured spectra. Adapted from [34].

Table 1. Results for the simulations of the EXAFS spectra at the Mn edge for the two configurations. Adapted from [34].

\begin{tabular}{lllll}
\hline & $N_{\mathrm{Ni}}$ & $R_{\mathrm{Ni}}(\AA)$ & $N_{\mathrm{Sb}}$ & $R_{\mathrm{Sb}}(\AA)$ \\
\hline Parallel & $3.98 \pm 0.24$ & $2.54 \pm 0.27$ & $6.01 \pm 0.30$ & $2.97 \pm 0.21$ \\
Perpendicular & $3.61 \pm 0.27$ & $2.55 \pm 0.28$ & $5.98 \pm 0.31$ & $2.95 \pm 0.21$ \\
Theory & 4 & 2.55 & 6 & 2.95 \\
\hline
\end{tabular}

spheres about $\mathrm{Mn}$ and in table 2 for the coordination spheres about $\mathrm{Ni}$, indicates that the short range atomic order is excellent. A small disagreement between calculated and experimental spectra in the parallel compared to the perpendicular configuration can be seen in figure 6 , especially near $k \cong 7 \AA^{-1}$. However, the resulting parameters shown in table 1 do not change significantly along the two directions, which suggests that possible lattice deformations, such as those induced by the substrate, are very weak. For Ni, the measured oscillations do not match expectations very well at small values of $k$ (characteristic of lon- 


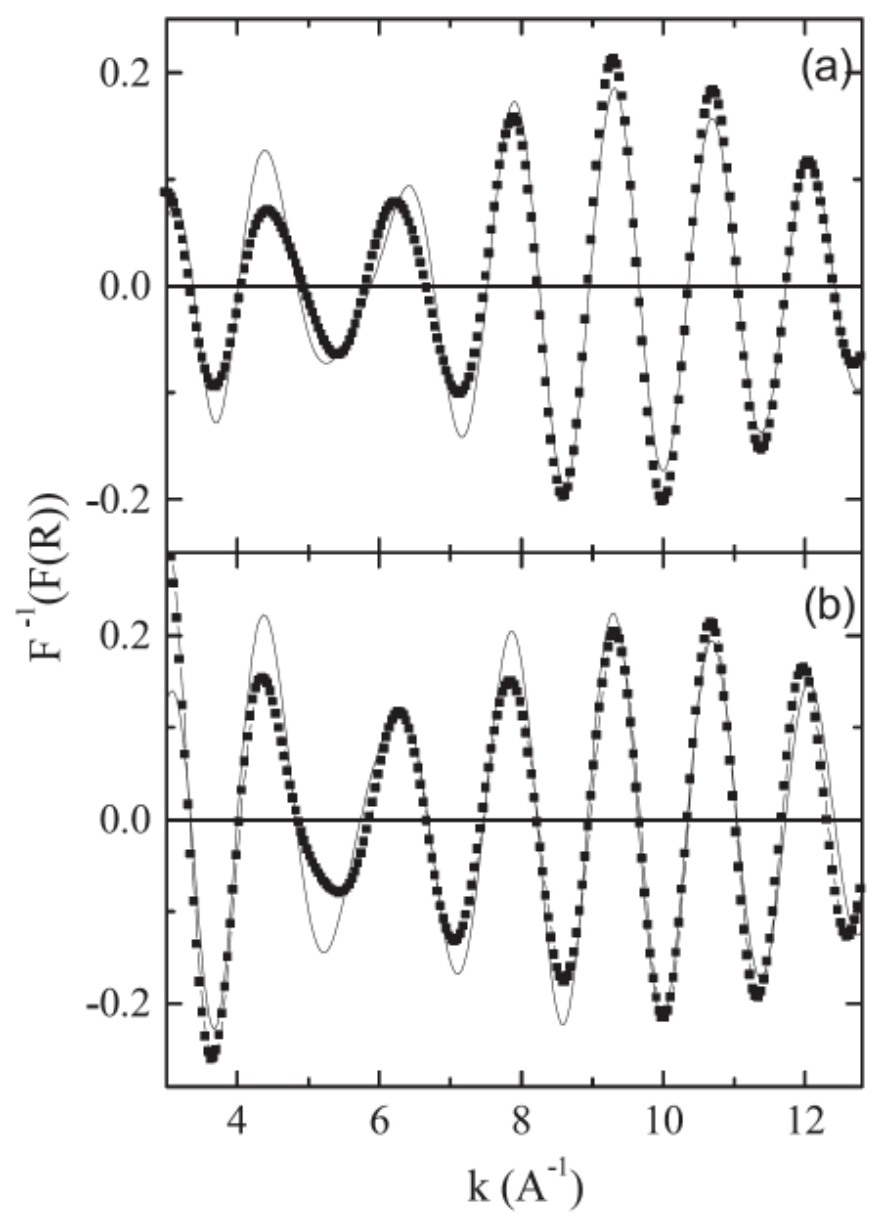

Figure 7. $k$-weighted EXAFS spectra recorded at the Ni edge in parallel (a) and perpendicular (b) configurations. The solid lines correspond to the simulations of the NiMnSb structure while the dots represent the experimental measured spectra. Adapted from [34].

Table 2. Results for the simulations of the EXAFS spectra at the Ni edge for the two configurations. Adapted from [34].

\begin{tabular}{lllll}
\hline & $N_{\mathrm{Mn}}$ & $R_{\mathrm{Mn}}(\AA)$ & $N_{\mathrm{Sb}}$ & $R_{\mathrm{Sb}}(\AA)$ \\
\hline Parallel & $3.88 \pm 0.47$ & $2.53 \pm 0.07$ & $3.51 \pm 0.59$ & $2.57 \pm 0.06$ \\
Perpendicular & $3.98 \pm 0.13$ & $2.54 \pm 0.20$ & $3.85 \pm 0.61$ & $2.56 \pm 0.17$ \\
Theory & 4 & 2.55 & 4 & 2.55 \\
\hline
\end{tabular}

ger distances), the fittings are better match in position and intensity for higher $k$ values, and the fit improves significantly. Note that the calculated spectra do not change shape and position when the $\mathrm{Mn}$ atoms and $\mathrm{Sb}$ atoms are interchanged in the first coordination shell about Ni.

To summarize, the EXAFS measurements showed that our NiMnSb films grown at $400{ }^{\circ} \mathrm{C}$ on Mo buffer layers exhibited good short range crystalline order, adding further support to the assertion that our $\mathrm{NiMnSb}(100)$ films are of high quality. The relatively elevated lattice mismatch of $5.8 \%$ between $\mathrm{NiMnSb}$ and Mo films is accommodated in the 

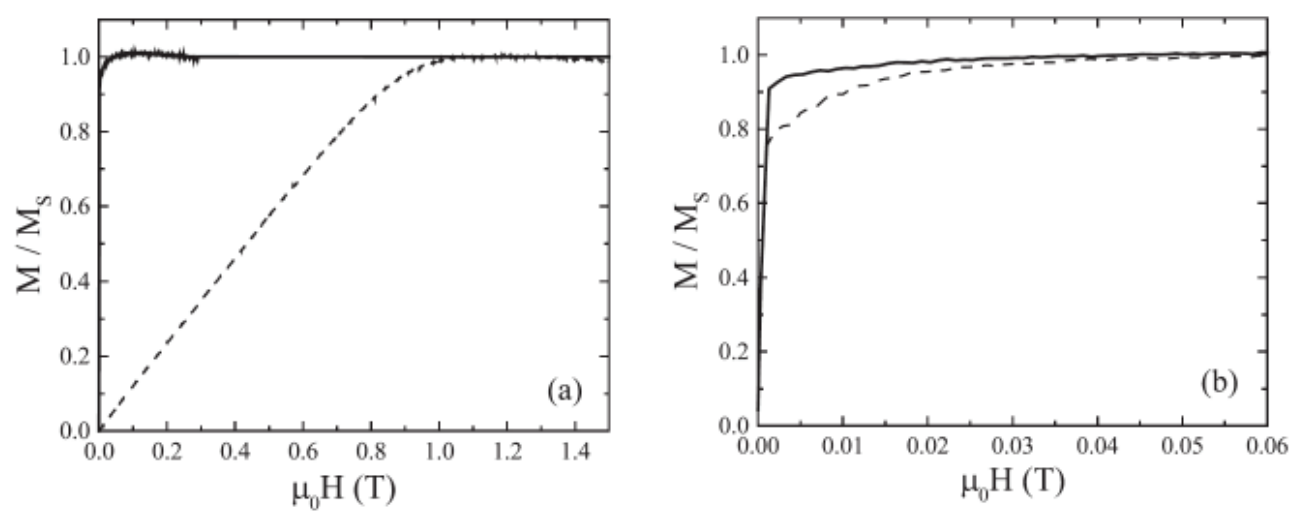

Figure 8. (a) $10 \mathrm{~K}$ virgin magnetization curves of an Mo $50 \AA$ / NiMnSb $1000 \AA /$ Mo 100 $\AA$ trilayer deposited in the optimal conditions of figure 5(d), with the applied field parallel (full line) and perpendicular (dotted line) to the film plane. (b) In-plane virgin magnetization curves with the applied field parallel to the (100) (full line) and (110) (dotted line). Adapted from [53].

first few monolayers of the NiMnSb layer, whose total thicknesses ranged from 500 to $1000 \AA$. The use of high quality thin films, rather than bulk single crystals is essential for the spin polarized electron spectroscopy measurements described later. Given the constraints necessary for careful surface characterization, transport measurements and polarization studies, only films grown under optimal conditions of figure 5(d) were used for the measurements described later.

\section{Magnetic and transport properties of the NiMnSb films}

Initial insight into the magnetic properties of crystalline NiMnSb films can be obtained from analysis of magnetic and transport properties, as a function of temperature. The magnetic measurements were measured with vibrating sample magnetometer (VSM) between 1.5 and $300 \mathrm{~K}$ and ferromagnetic resonance measurements were performed at RT on a standard $\mathrm{X}$ band Bruker spectrometer. Transport measurements were also undertaken between 1.5 and $300 \mathrm{~K}$ in the conventional AC four-point probe geometry on the $\mathrm{NiMnSb}$ films with typical thicknesses of 500-1000 A.

The virgin magnetization curves of a $1000 \AA$ thick film, measured at $10 \mathrm{~K}$ in an applied field, with the parallel and perpendicular components presented in figure 8 . The magnetization clearly lies in the film plane with an out of plane anisotropy of $0.9 \mathrm{~T}$. Assuming that this anisotropy is due solely to shape anisotropy $\left(H_{\mathrm{A}}=4 \pi M_{\mathrm{s}}\right)$, then a magnetic moment per Mn atom of $3.9 \pm 0.2 \mu_{\mathrm{B}}$ is deduced. This value is consistent with the expected value of $4.0 \mu_{\mathrm{B}}$ per formulae unit determined from ground state band structure calculations [1] and with the value measured on bulk single crystals by polarized neutron diffraction (3.98 $\pm 0.03 \mu_{\mathrm{B}}$ per formulae unit [48], as discussed later). Regrettably, this does not prove half-metallic character as this is not a zero-field measurement, and an increase in spin wave stiffness is expected with high magnetic fields [37,60]. Also the uncertainly in the measurement does not make it possible to exclude correlation effects (i.e. a non-quasiparticle density of states) $[61,62]$ that can limit polarization from reaching $100 \%$ in a small way in the ground state.

The in-plane magnetic anisotropy was investigated both by conventional magnetometry (VSM) and by ferromagnetic resonance. As a fourfold symmetry is expected in cubic crystals, the virgin magnetization curves were measured with the field along the (100) and (110) crystallographic directions (figure 8(b)). The (110) axis appears to be the hard axis 


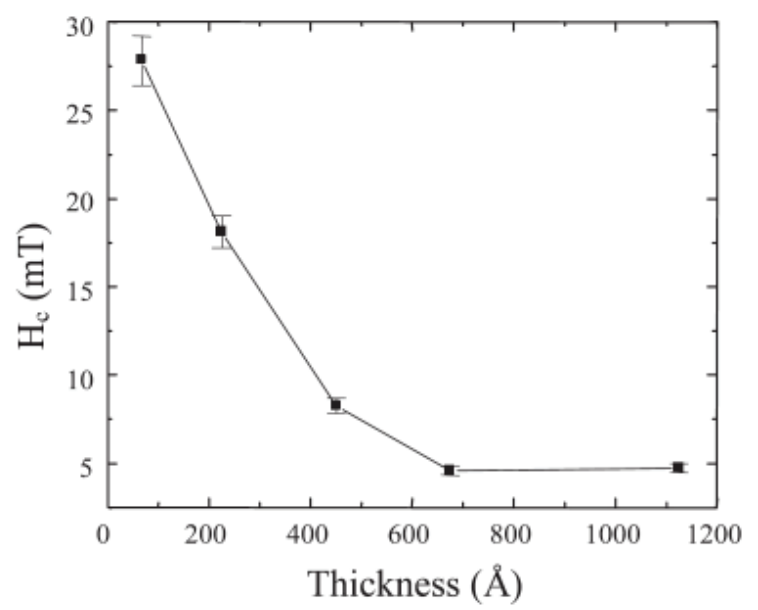

Figure 9. Evolution of the coercive field as a function of film's thickness, at room temperature. Adapted from [53].

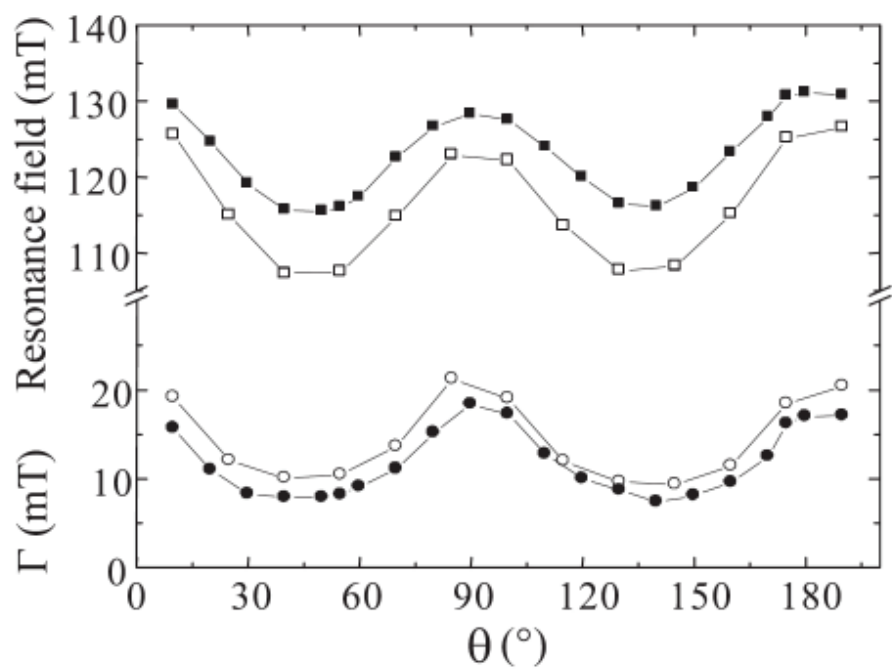

Figure 10. Resonance field (top) and linewidth (bottom) as a function of angle $\theta$ between the applied field and the (110) axis, at $300 \mathrm{~K}$ (full symbols) and at $10 \mathrm{~K}$ (empty symbols). Adapted from [34].

with a corresponding anisotropy field of $25 \pm 2 \mathrm{mT}$. The coercive field, shown in figure 9, varies from 5 to $30 \mathrm{mT}$, in an inverse relationship with the film thickness - the thinner the film, the bigger the coercive field, a common phenomena in thin ferromagnetic films which can occur due to trapping of the coercive field by the surface imperfections or by surface oxidation as the layer thickness gets very small.

The evolution of the FMR resonance field as a function of the in-plane angle $\theta$ with the (110) axis is summarized in figure 10, for films at both $300 \mathrm{~K}$ (filled symbols) and at $10 \mathrm{~K}$ (open symbols). The (100) easy axis and the fourfold anisotropy are clearly evident, with a deduced anisotropy field of $20 \pm 2 \mathrm{mT}$ at $10 \mathrm{~K}$, in good agreement with the value obtained from static measurements, of $25 \mathrm{mT}$ also at $10 \mathrm{~K}$. The linewidth follows 


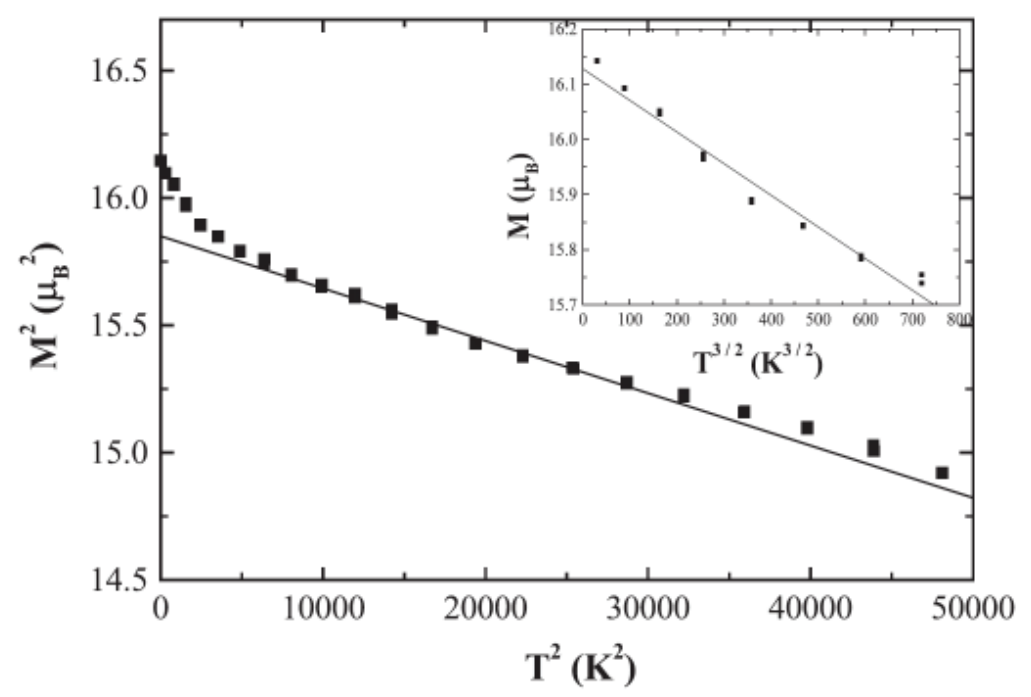

Figure 11. Temperature dependence of the magnetization for $T>100 \mathrm{~K}$ and as an inset, for $T<100 \mathrm{~K}$. Adapted from [53].

the same angular dependence as the resonance field, with small overall values (10-20 mT) which indicates good magnetic homogeneity.

The temperature dependence of the magnetization along the (110) axis below $200 \mathrm{~K}$ is illustrated in figure 11 . Above $100 \mathrm{~K}, M^{2}$ varies linearly as a function of $T^{2}$, whereas a deviation from the $T^{2}$ law appears for temperatures lower than $100 \mathrm{~K}$, where the magnetization varies more rapidly $\left(\sim T^{3 / 2}\right)$. Such a behavior is identical to what has been observed in bulk single crystals and is ascribed to a transition from a low temperature localized ferromagnetism in which collective spin wave excitations predominate (as expected for a nominal half-metal or very high polarization ferromagnet) to a high temperature itinerantlike ferromagnetism in which spin fluctuations contribute to the decay of the net moment [51]. The very existence of spin waves at lower temperatures, assuming the model [51] is correct, precludes ideal (100\% polarization) half-metallic character $[24,45]$.

The temperature dependence of the resistivity, after the subtraction of the Mo buffer layer contribution, is shown in figure 12, again for a $1000 \AA \mathrm{NiMnSb}$ epitaxial film deposited in the optimal conditions described in section 2 . The extrapolated residual resistivity is $5.3 \mu \Omega \mathrm{cm}$, which is lower than the best values reported for polycrystalline thin films $(\sim 20 \mu \Omega \mathrm{cm})$ and polycrystalline bulk samples $(6.9 \mu \Omega \mathrm{cm})$ [63], and consistent with the high quality of our films. A kink in the temperature dependence of the resistivity occurs around $70 \mathrm{~K}$, which has been already observed in NiMnSb polycrystalline film and bulk samples $[51,63,64]$. For temperatures lower than $70 \mathrm{~K}$ the resistivity obeys a $T^{2}$ law: $R(T)=R_{0}+A T^{2}$ with $R_{0}=5.3 \mu \Omega \mathrm{cm}$ and $A=5.8 \times 10^{-4} \mu \Omega \mathrm{cm} \mathrm{K}^{-2}$, while at higher temperatures $R(T)=R_{0}^{\prime}+A^{\prime} T^{1.65}$ with $R_{0}^{\prime}=6.1 \mu \Omega \mathrm{cm}$ and $A^{\prime}=1.7 \times 10^{-3} \mu \Omega \mathrm{cm} \mathrm{K} K^{-1.65}$. As it is more and more difficult to extract the resistivity of the NiMnSb layer in the Mo/ $\mathrm{NiMnSb} / \mathrm{Mo}$ trilayer when its thickness is reduced (the parallel resistor model is no longer strictly valid), we have plotted the room temperature resistance versus the inverse of the film thickness (figure 13). A linear behavior is observed which indicates a thickness independent resistivity in the thickness range studied here (100-1000 $\AA$ ). The slope of the linear fit yields the average room temperature NiMnSb resistivity of $19.9 \pm 1.5 \mu \Omega \mathrm{cm}$. 


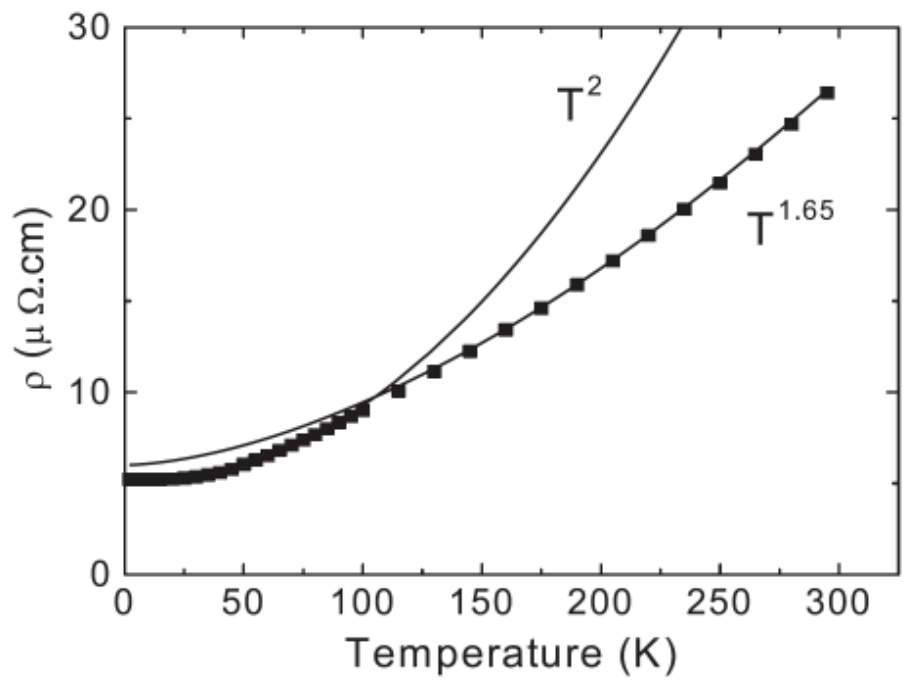

Figure 12. The thermal variation of the resistivity for a $1000 \AA$ thick NiMnSb film. Adapted from [34, 53].

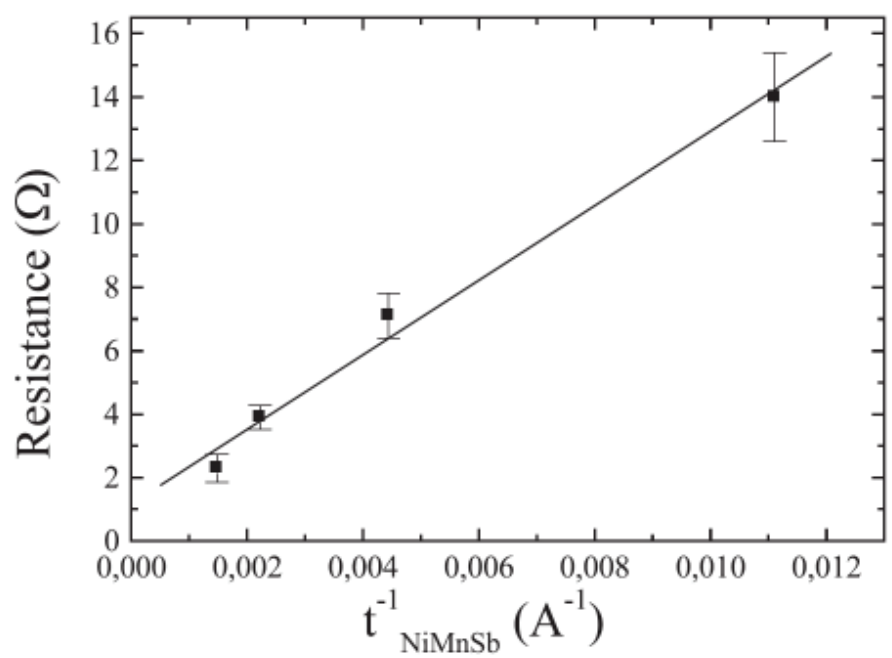

Figure 13. Thickness dependence of the overall resistance in $\mathrm{Mo} / \mathrm{NiMnSb}(\mathrm{t}) / \mathrm{Mo}$ trilayers deposited in the optimal conditions of figure 5(d). Adapted from [53].

\section{Characterization of the surface composition}

The question "what is a surface" can be answered in many ways depending on the significance of the property studied. In a strictly physical sense, it is the outermost, top monatomic layer of a solid. Since it is now well known that equilibrium segregation can exist at the surface or in some cases extending to a many atomic layers away from the surface, the study of surface composition is necessary in any compound or alloy system before one can assess the value of spin polarization. We adopted angle resolved x-ray photoemission (ARXPS) as the most expedient technique for characterizing the surface composition of half-metals and related compounds. 


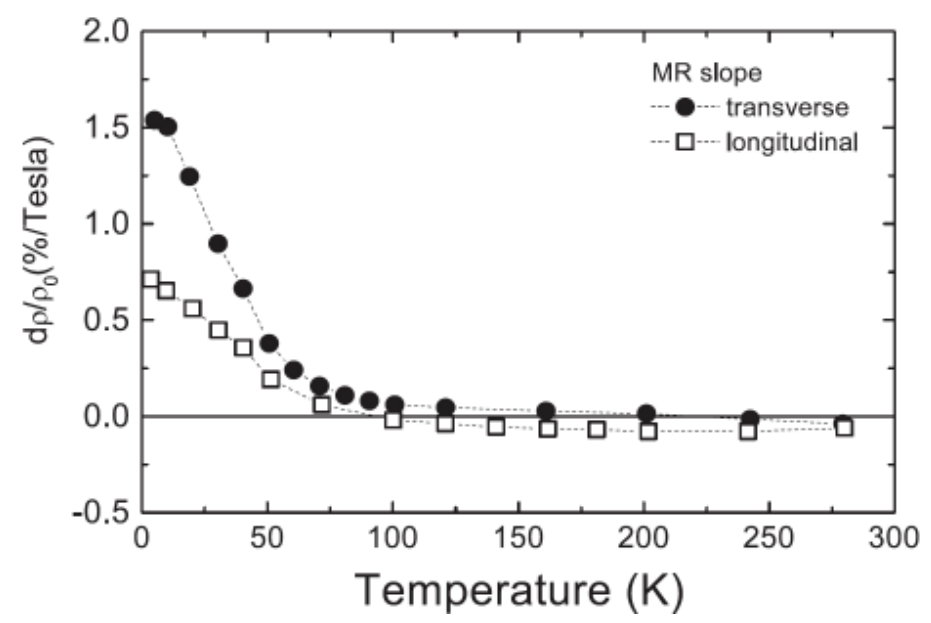

Figure 14. Transverse and longitudinal MR in NiMnSb films (slopes of MR curves between 1 and 6 T). Adapted from [52].

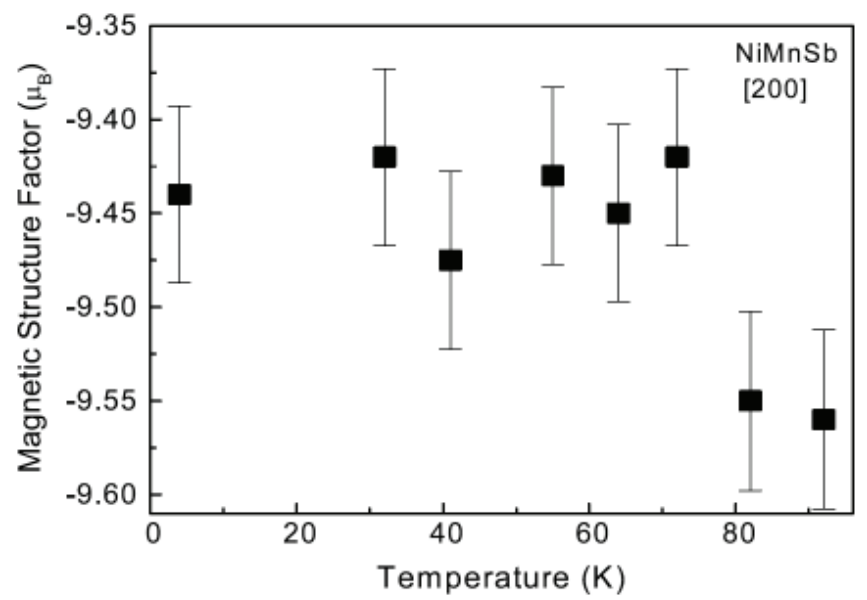

Figure 15. The temperature dependence of the magnetic structure factor for the $\langle 200\rangle$ peak obtained from neutron diffraction. Adapted from [33, 59].

To excite the core level photoelectrons, we used an x-ray source with an Mg anode having the main $\mathrm{Mg} \mathrm{K}_{\alpha 1,2}$ line at $1253.6 \mathrm{eV}$ with the full width at half-maximum of 0.65 $\mathrm{eV}$. We used a hemispherical electron energy analyzer from Physical Electronics (PHI Model 10-360 Precision Energy Analyzer), with 8.5 inch radius. The combined resolution of the x-ray source and analyzer was $650 \mathrm{meV}$.

In ARXPS, the surface composition can be determined with great accuracy since the effective probing depth becomes shorter as the emission angle is increased with respect to the surface normal. The experimental core level intensities for any two components from a multicomponent alloy are acquired at several emission angles $\theta$, usually from $\theta=0^{\circ}$ (normal emission) to $60^{\circ}$ (off-normal emission). Then, a linear background contribution is systematically subtracted from each raw spectrum. The peak intensities are further normalized by the corresponding differential cross section for emission and by the analyzer transmission function [48, 65-68]. The experimentally normalized intensity ratio for any 
two elements $\mathrm{A}$ and $\mathrm{B}$ is thus given by:

$$
R^{\exp }(\theta)=\frac{I(\mathrm{~A}) / \sigma_{\mathrm{A}}}{I(\mathrm{~B}) / \sigma_{\mathrm{B}}} \frac{T\left(E_{\mathrm{A}}\right)}{T\left(E_{\mathrm{B}}\right)}
$$

where $I(\mathrm{~A})$ and $I(\mathrm{~B})$ are the measured core level intensities for elements $\mathrm{A}$ and $\mathrm{B}, \sigma_{\mathrm{A}}$ and $\sigma_{\mathrm{B}}$ are the cross sections, and $T\left(E_{\mathrm{A}}\right)$ and $T\left(E_{\mathrm{B}}\right)$ are the transmission functions of the analyzer for elements $\mathrm{A}$ and $\mathrm{B}$ as a function of the corresponding photoelectron kinetic energies $E_{\mathrm{A}}$ and $E_{\mathrm{B}}$ and are based on the measured transmission functions for each analyzer. For example, in our case, the transmission function of PHI 10-360 Precision Energy Analyzer is $T\left(E_{\mathrm{A}}\right)={\sqrt{E_{\mathrm{A}}}}[69]$.

Over the past decades, the phenomenon of surface segregation attracted quite a lot of theoretical interest. Up to now, various theories have been developed in order to account for the enrichment at the binary alloy surface. The first-principles approach [70], embedded atom method (EAM) model [71], Finnis-Sinclair (FS) potential [72], and BozzoloFerrante-Smith (BFS) method [73] have been used to simulate the surface segregation for fcc type random and ordered alloys, while for fcc and hep elements the modified analytic embedded atom method (MAEAM) many-body potential is used to theoretically study the surface segregation phenomena of binary alloys [74]. Unfortunately, to our knowledge, there are no detailed theoretical studies of the surface segregation of multicomponent half-metal alloys, although there are now some studies of the NiMnSb(100) surface stability $[38,42,44,75,76]$. We use here a simplified model to fit the experimental intensity ratios obtained from ARXPS.

The comparison between theory and experiment is accomplished through considerations of the theoretical normalized intensity ratio of element $\mathrm{A}$ and $\mathrm{B}$ as given by:

$$
R^{\text {theor }}(\theta)=\frac{\sum_{j=0}^{\infty} f_{j}(\mathrm{~A}) \exp \left[\frac{-\mathrm{j} d}{\lambda_{\mathrm{A}}^{j} \cos (\theta)}\right]}{\sum_{j=0}^{\infty} f_{j}(\mathrm{~B}) \exp \left[\frac{-\mathrm{j} d}{\lambda_{\mathrm{B}}^{j} \cos (\theta)}\right]}
$$

where $\lambda_{\mathrm{A}}^{j}$ and $\lambda_{\mathrm{B}}^{j}$ are the inelastic mean free paths of the core electrons generated from elements $\mathrm{A}$ and $\mathrm{B}$ respectively and passing through the material contained in layer $j$. The inelastic mean free paths can be adopted from previously published methodologies [77] contained within the NIST Electron Inelastic Mean Free Path program [78]. The atomic fraction of element A (chosen as the element which segregates to the surface) in the $j$ th layer below the surface is given by:

$$
f_{j}(\mathrm{~A})=b+\delta \exp (-\mathrm{j} d / G)
$$

where $b$ is the bulk fraction of element A, $\delta$ and $G$ are fitting parameters representing the extent of the segregation and the segregation depth respectively, and $d$ is the distance between atomic layers. These two quantities are also the fitting parameters when comparing the model with experimental values. From the profile form $f_{j}(\mathrm{~A})$ one can calculate the apparent surface concentration (or relative intensity) of element A for a particular core level.

Considering now the case of the NiMnSb films, three sublattices correspond to a given atom respectively $(\mathrm{Ni}, \mathrm{Mn}$ and $\mathrm{Sb}$ ) whilst the fourth is empty. In this crystallographic structure, the (001) surface of $\mathrm{NiMnSb}$ is binary, composed of alternate planes of $\{\mathrm{Mn}, \mathrm{Sb}\}$ and $\{\mathrm{Ni}$, vacancy $\}$. If the surface free energy is different from the bulk, substantial segregation may appear on the surface, disturbing both the crystalline and electronic structures in that region.

The NiMnSb epitaxial films were protected with a $1000 \AA$ of Sb capping layer to prevent oxidation and surface disorder. The Sb layers were removed in UHV, without destroying the NiMnSb surface, by application of a "flash" thermal treatment which resulted in rapid $\mathrm{Sb}$ sublimation. Moreover, this treatment limits the possible thermal activated segregation to a minimum. The thermal treatment involved resistive heating of the sample 


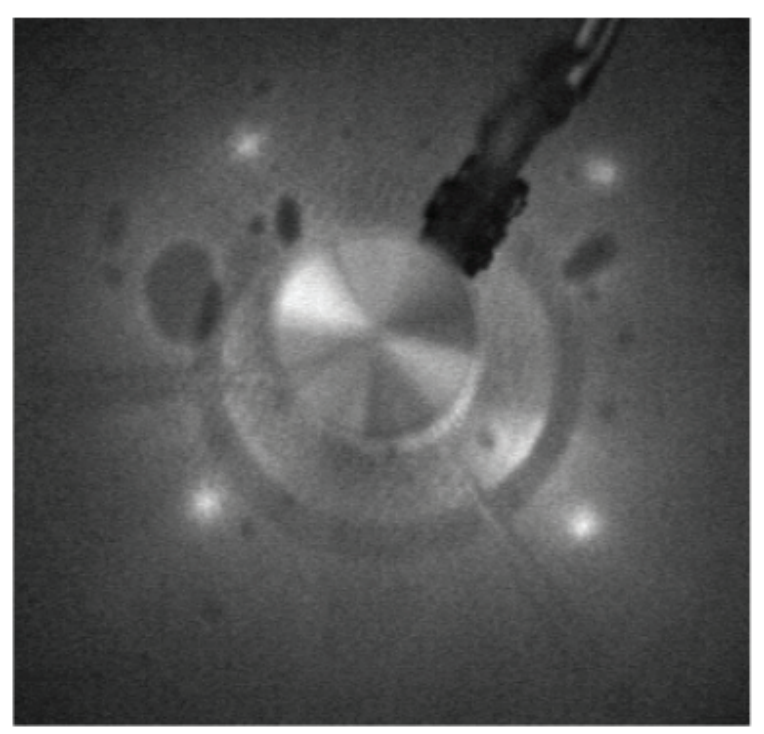

Figure 16. The LEED pattern of a stoichiometric ordered NiMnSb surface obtained through annealing the sample to $400{ }^{\circ} \mathrm{C}$. Adapted from [32].

up to $400{ }^{\circ} \mathrm{C}$ for several minutes, followed by a fast cooling to room temperature. No evidence of surface $\mathrm{Sb}$ diffusion into the NiMnSb bulk was found in the angle resolved XPS surface characterization studies described below and such Sb diffusion is unlikely in any case due to reduced melting temperature of $\mathrm{Sb}$ compared to $\mathrm{NiMnSb}$. Once the $\mathrm{Sb}$ capping layer was removed, the crystallinity and orientation of the stoichiometric $\mathrm{NiMnSb}(100)$ surface was again established using low energy electron diffraction (LEED). Figure 16 shows a LEED image recorded at room temperature, using $34 \mathrm{eV}$ incident electron energy, for the stoichiometric surface obtained after removal of the Sb capping layer.

The fourfold low energy electron diffraction images exhibited very sharp spots, indicative of an ordered surface region. Knowing the geometry and dimensions of the LEED system, the surface lattice constant was extracted from equation:

$$
2 a \sin \theta=n \lambda, \quad \lambda=\frac{h^{2}}{\sqrt{2 m E_{\mathrm{kin}}}}
$$

where $n=\sqrt{h^{2}+k^{2}}$ for a square lattice, $E_{\text {kin }}$ is the kinetic energy of the incident electrons, and $\sin \theta$ can be calculated from the geometry of the measurement. The distance between the central spot (not shown in figure 16) and any of the fourfold-symmetric spots corresponds to the $\mathrm{Mn}-\mathrm{Mn}$ or equivalently, $\mathrm{Sb}-\mathrm{Sb}$ distance of $4.24 \AA$ in the real space. The surface thus prepared can be considered a proper termination of the bulk NiMnSb, consistent with the angle resolved XPS described below. The lifetime of the surface is limited by carbon contamination to several hours.

The terminal layer of the thin film was determined using angle resolved photoemission and the fitting procedures introduced above. The core levels with the highest cross section for each atomic species have been monitored at room temperature $-2 p_{1 / 2}$ and $2 p_{3 / 2}$ for $\mathrm{Mn}, 3 \mathrm{~d}_{3 / 2}$ and $3 \mathrm{~d}_{5 / 2}$ for $\mathrm{Sb}, 2 \mathrm{p}_{3 / 2}$ for Ni. Surface contributions to the peak intensities are enhanced at high emission angles, whereas bulk contributions dominate at normal $\left(0^{\circ}\right.$ with respect to the surface normal) emission, as can be seen in figure 17. The angular resolution of the acquired ARXPS spectra was $2^{\circ}$, smaller than the acceptance angle of the 


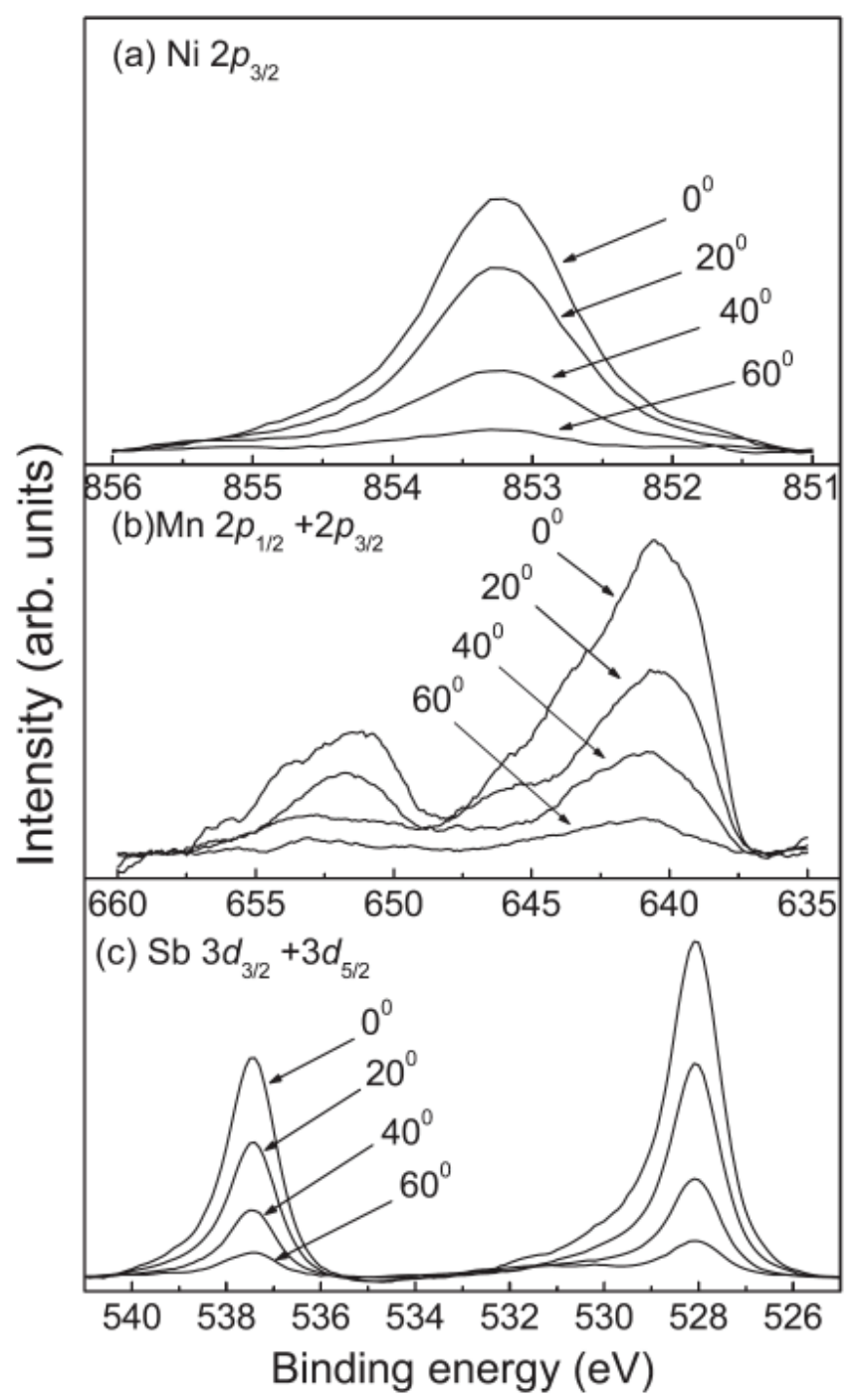

Figure 17. The Ni $2 \mathrm{p}_{3 / 2}$ (a), Mn $2 \mathrm{p}_{3 / 2}+2 \mathrm{p}_{1 / 2}$ (b), and Sb $3 \mathrm{~d}_{5 / 2}+3 \mathrm{~d}_{3 / 2}$ (c) core levels of $\mathrm{NiMnSb}$ are shown as a function of emission angle. Emission along the surface normal is the spectrum labeled with $0^{\circ}$. Adapted from [48].

analyzer. The first observation that can be drawn from this figure refers to the shape of all spectra, which are very well defined and free of satellites. The presence of satellites near the main photoemission peaks would be indicative of either strong core-hole interactions (as is the case in CMR perovskites [66]) or the presence of more than one equivalent crystallographic site for the same atomic species.

The increase in the Mn 2 $\mathrm{p}_{3 / 2}$ halfwidth is consistent with the placement of $\mathrm{Mn}$ in a more metallic environment in half-metallic NiMnSb than is the case in the half-metallic manganese perovskites, as well as because of the multiplicity of the final states possible, including a surface to bulk core level shift, and has been observed before for other cubic compound pnictide systems [79]. Concerning the core level peak positions, the binding energy corresponding to each core level matches the values reported in the literature [80], as summarized in table 3 . 


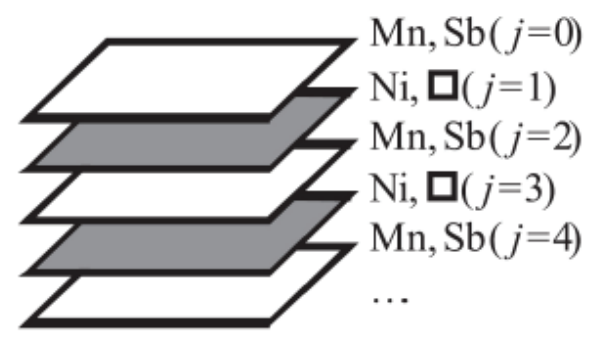

Figure 18. Layer stacking sequence assumed for the stoichiometric surface.

Table 3. Comparison between the binding energies obtained experimentally and the values published in [80].

\begin{tabular}{|c|c|c|c|c|c|}
\hline & $\mathrm{Ni} 2 \mathrm{p}_{3 / 2}(\mathrm{eV})$ & $\mathrm{Mn} 2 \mathrm{p}_{3 / 2}(\mathrm{eV})$ & $\mathrm{Mn} 2 \mathrm{p}_{1 / 2}(\mathrm{eV})$ & $\mathrm{Sb} 3 \mathrm{~d}_{5 / 2}(\mathrm{eV})$ & $\mathrm{Sb} 3 \mathrm{~d}_{3 / 2}(\mathrm{eV})$ \\
\hline Exp. values & $853.2 \pm 0.1$ & $640.1 \pm 0.1$ & $651.0 \pm 0.1$ & $528.1 \pm 0.1$ & $537.5 \pm 0.1$ \\
\hline Reference $[80]$ & 853.3 & 639.4 & 650.6 & 528.2 & 537.7 \\
\hline
\end{tabular}

At last, it is important to note that, apart from Mn, the positions of the photoelectron peaks in figure 17 do not depend on emission angle, which indicates that the valence state of the atoms in the surface is the same as the chemical environment deeper in the bulk, neglecting issues related to the surface to bulk core level shift associated with the Mn $2 p$ which suggest that Mn occupies the surface terminal layer. We can determine the surface stoichiometry by fitting specific angle resolved XPS intensity ratios with a theoretical model based on the naturally layered structure of NiMnSb. As indicated in figure 18, we start by assuming that the Heusler alloy structure consists of alternating layers of $\{\mathrm{Mn}$, $\mathrm{Sb}\}$ and $\{\mathrm{Ni}$, vacancy $\}$, spaced at a distance of $1.5 \AA$. The stoichiometric crystal must be terminated by either a $\{\mathrm{Mn}, \mathrm{Sb}\}$ or a $\{\mathrm{Ni}$, vacancy $\}$ plane. We also considered the possibilities of atomic disorder in the $\mathrm{Ni}$ vacancy planes resulted from the replacement of the vacancy site by $\mathrm{Mn}, \mathrm{Sb}$ or Ni.

Different core level intensity ratios are plotted against emission angle in figure 19. Each raw spectrum was analyzed by first subtracting a linear background and then normalizing with the corresponding cross section values from [81], as well as with the analyzer transmission function. The model calculations presented as lines in figure 19 are obtained from summing over each layer contributing to the photoemission signal. This can be translated by attributing an index $j$ for each atomic plane and then summing over odd or even values of $j$ in equation (4). The experimental data in figure 19 are compared to several models for the surface termination including (I) $\{\mathrm{MnSb}\}$ (surface) $/ \mathrm{Ni}$, vacancy/Mn, $\mathrm{Sb}]_{n}$ which represents the case of a perfect ordered crystal, and (II) $\{\mathrm{Ni}, \mathrm{Mn}\}$ (surface)/ $[\mathrm{Mn}, \mathrm{Sb} / \mathrm{Ni} \text {, vacancy }]_{n}$ and (III) $\{\mathrm{Ni}, \mathrm{Sb}\}$ (surface) $/ \mathrm{Mn}, \mathrm{Sb} / \mathrm{Ni}$, vacancy $]_{n}$, which represent the case of the replacement of the vacancy site by a $\mathrm{Mn}$ or $\mathrm{Sb}$ atom, respectively.

For example, in model (I), the $\mathrm{Mn}$ and $\mathrm{Sb}$ atoms are assumed to be in even layers ( $j=$ even), while the Ni atom is in odd layers $(j=$ odd $)$. The elemental ratios can be written as:

$$
\begin{aligned}
& \frac{I(\mathrm{Mn})}{I(\mathrm{Sb})}=\frac{\sum_{j=0}^{\infty} 0.5 \exp \left(-\frac{2 \mathrm{j}}{\lambda_{\mathrm{Mn}} \cos \theta}\right)}{\sum_{j=0}^{\infty} 0.5 \exp \left(-\frac{2 \mathrm{j}}{\lambda_{\mathrm{Sb}} \cos \theta}\right)}=\frac{1-\exp \left(-\frac{2}{\lambda_{\mathrm{Sb}} \cos \theta}\right)}{1-\exp \left(-\frac{2}{\lambda_{\mathrm{Mn}} \cos \theta}\right)}, \\
& \frac{I(\mathrm{Ni})}{I(\mathrm{Sb})}=\frac{\sum_{j=0}^{\infty} 0.5 \exp \left(-\frac{2 j+1}{\lambda_{\mathrm{Ni}} \cos \theta}\right)}{\sum_{j=0}^{\infty} 0.5 \exp \left(-\frac{2 \mathrm{j}}{\lambda_{\mathrm{Sb}} \cos \theta}\right)}=\exp \left(-\frac{1}{\lambda_{\mathrm{Ni}} \cos \theta}\right) \frac{1-\exp \left(-\frac{2}{\lambda_{\mathrm{Sb}} \cos \theta}\right)}{1-\exp \left(-\frac{2}{\lambda_{\mathrm{Ni}} \cos \theta}\right)},
\end{aligned}
$$




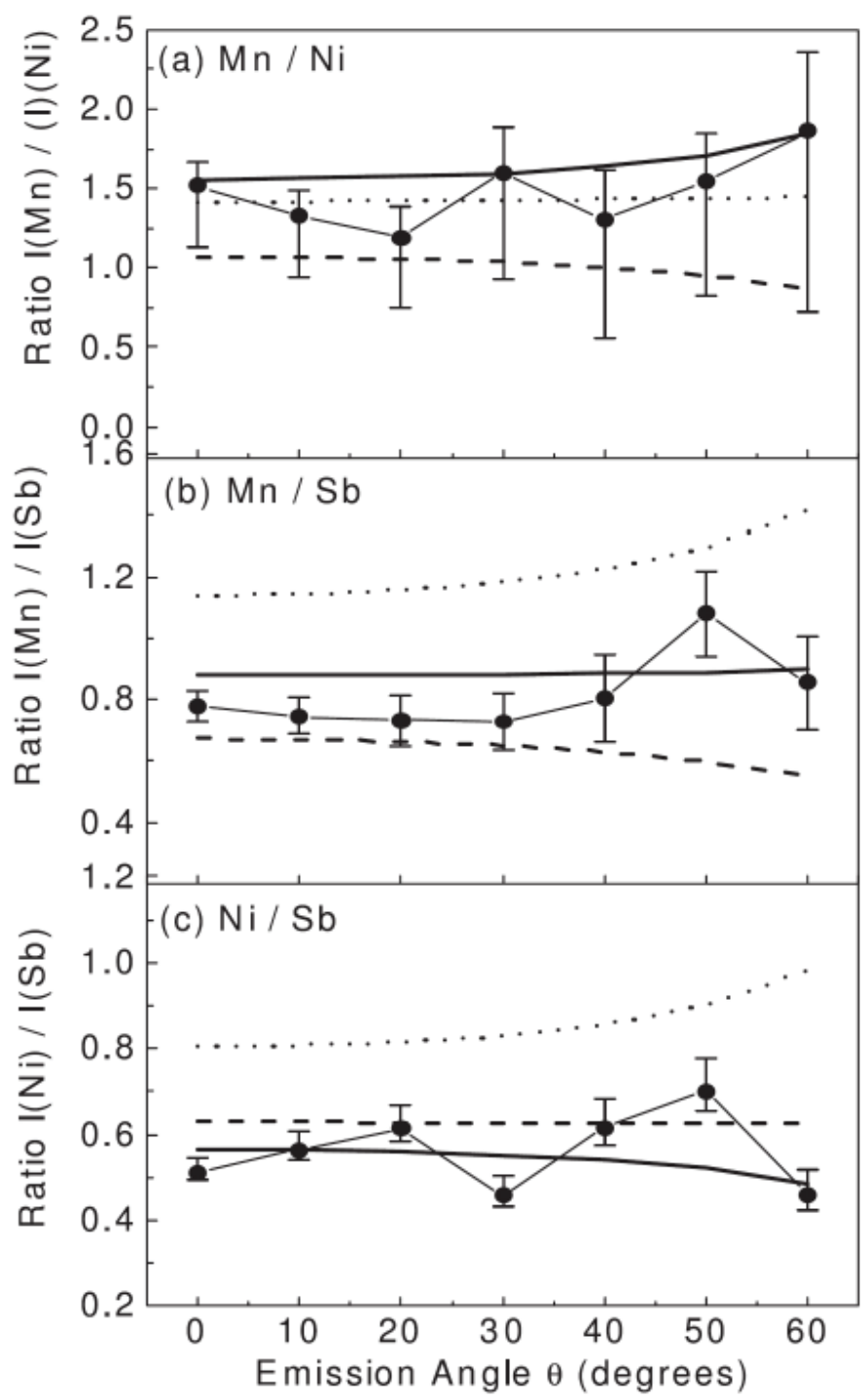

Figure 19. Angle resolved photoemission intensities ratios of the Ni $2 p_{3 / 2}$, Mn $2 p_{3 / 2}+$ $2 \mathrm{p}_{1 / 2}$, and $\mathrm{Sb} 3 \mathrm{~d}_{5 / 2}+3 \mathrm{~d}_{3 / 2}$ core levels of NiMnSb following the removal of excess $\mathrm{Sb}$ with a $700 \mathrm{~K}$ flash anneal (circles). The data are compared to several models for surface termination including the stoichiometric alloy $\{\mathrm{MnSb}\}$ (surface)/[Ni, vacancy/Mn, $\mathrm{Sb}]_{n}$ (I continuous line), $\{\mathrm{Ni}, \mathrm{Mn}\}$ (surface)/[Mn, $\mathrm{Sb} / \mathrm{Ni}$, vacancy $]_{n}$ (II dotted line), and $\{\mathrm{Ni}$, $\mathrm{Sb}\}$ (surface)/[Mn, $\mathrm{Sb} / \mathrm{Ni}$, vacancy $]_{n}$ (III dashed line). Adapted from $[32,48]$.

$\frac{I(\mathrm{Mn})}{I(\mathrm{Ni})}=\frac{\sum_{j=0}^{\infty} 0.5 \exp \left(-\frac{2 \mathrm{j}}{\lambda_{\mathrm{Mn}} \cos \theta}\right)}{\sum_{j=0}^{\infty} 0.5 \exp \left(-\frac{2 j+1}{\lambda_{\mathrm{Ni}} \cos \theta}\right)}=\exp \left(\frac{1}{\lambda_{\mathrm{Ni}} \cos \theta}\right) \frac{1-\exp \left(-\frac{2}{\lambda_{\mathrm{Ni}} \cos \theta}\right)}{1-\exp \left(-\frac{2}{\lambda_{\mathrm{Mn}} \cos \theta}\right)}$,

where the $\lambda \mathrm{s}$ are the effective mean free paths corresponding to each core level measured, and $\theta$ is the emission angle. The other two models (II and III) shown in figure 19 assume $\mathrm{Ni}$ atoms in the even layers ( $j=$ even) and $\mathrm{Mn} / \mathrm{Sb}$ in the odd layers ( $j=$ odd). The fits resulted from these three models give poor agreement with the measured intensity ratios. The best fit, shown as a continuous line in figure 19, was obtained by the model with a $\{\mathrm{Mn}, \mathrm{Sb}\}($ surface $) / \mathrm{Ni}$, vacancy/Mn, $\mathrm{Sb}]_{n}$ layered structure. 


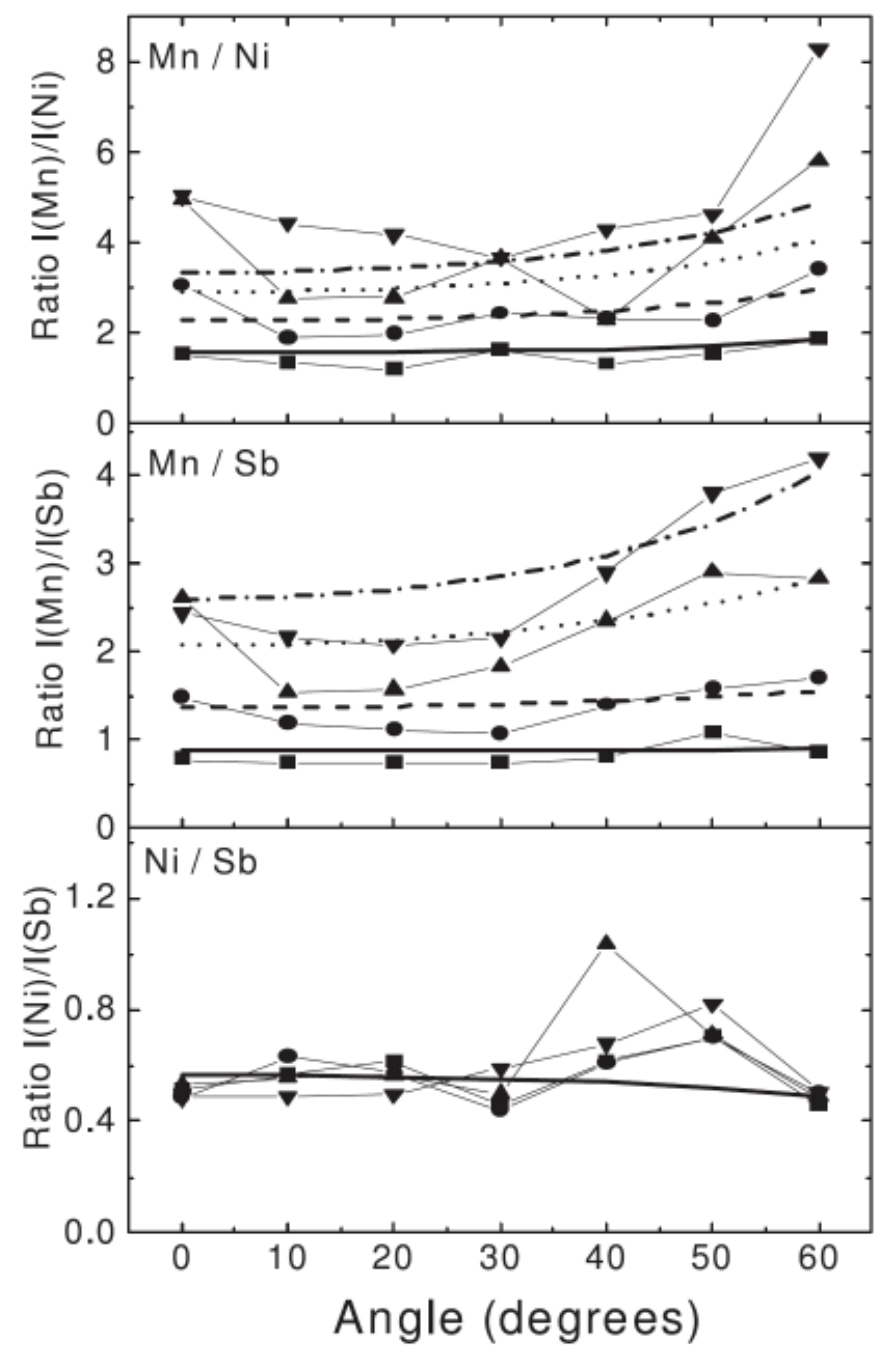

Figure 20. The angle resolved $x$-ray photoemission intensity ratios of the $\mathrm{Ni} 2 \mathrm{p}_{3 / 2}$, Mn $2 \mathrm{p}_{3 / 2}$, and $\mathrm{Sb} 3 \mathrm{~d}_{5 / 2}$ core levels of NiMnSb. The data are compiled from spectra taken as a function of emission angle following removal of the excess Sb with a $700 \mathrm{~K}$ anneal (squares) and following continued sputtering and annealing cycles of $10 \mathrm{~min}$ (circles) followed by $1 / 2 \mathrm{~h}$ (up-triangles) and finally by $1 \mathrm{~h}$ (down-triangles) total annealing times. The data points are fitted with a segregation model in which Mn atoms migrate to both vacancy sites as well as replace $\mathrm{Sb}$ and $\mathrm{Ni}$ atoms from their lattice sites in the surface region except for data for the surface stoichiometric alloy termination $\mathrm{Sb}$ (surface): $\mathrm{Ni}$ :(MnSb: $\mathrm{Ni})_{n}$ shown with continuous line. Adapted from [48].

The electron mean free paths used in the above equations are adapted from calculations of Penn in [82]. The approximation needed in calculating the $\lambda$ s is dictated by the fact that each photoelectron must travel through a selvedge region with unknown relative density before escape into vacuum. We considered an average value for the mean free path of each element monitored, as calculated in table 4 . For example, a Mn 2 $\mathrm{p}_{3 / 2}$ photoelectron, at $613 \mathrm{eV}$ kinetic energy, has a mean free path of $8.76 \AA$ in a Ni host, and 11.65 $\AA \AA$ in a Mn host material, $6.49 \AA$ in a Sb host. The Mn photoelectrons mean free path used in the above sums is the average value of $\lambda_{\text {avg }}^{\mathrm{Mn}}=9.48 \AA$. 
Table 4. Approximation of the theoretical escape depths for the Mn, Ni and Sb, obtained by taking the average of the respective impurity mean free paths in different pure host materials.

\begin{tabular}{llllllll}
\hline Element & $E_{\text {kin }}(\mathrm{eV})$ & $a$ & $b$ & $\lambda$ in $\mathrm{Mn}(\AA)$ & $\lambda$ in Ni $(\AA)$ & $\lambda$ in Sb $(\AA)$ & $\lambda_{\text {avg }}(\AA)$ \\
\hline $\mathrm{Mn}$ & 613 & 19.7 & -2.87 & 8.76 & 8.05 & 11.65 & $\mathbf{9 . 4 8}$ \\
$\mathrm{Ni}$ & 399.5 & 23.7 & -3.21 & 6.49 & 6.06 & 8.45 & $\mathbf{6 . 9 9}$ \\
$\mathrm{Sb}$ & 716 & 12.4 & -2.18 & 9.81 & 8.99 & 13.15 & $\mathbf{1 0 . 6 5}$ \\
\hline
\end{tabular}

As mentioned above, the lifetime of an ordered stoichiometric NiMnSb surface, at room temperature, is only approximately several hours, even in ultrahigh vacuum conditions. After performing the same cleaning procedure for three similar samples, we have observed that continuing the thermal treatments at $400{ }^{\circ} \mathrm{C}$ for more than several minutes resulted in activation of surface segregation. LEED images showed that the segregated surface remained ordered, with the same lattice constant of $6 \AA$. Only after the analysis of the angle resolved XPS intensity ratios, shown in figure 20, revealed a substantial deviation from the perfect bulk termination surface stoichiometry, which is expected from other studies as well [31].

Qualitatively, figure 20 shows that both $\mathrm{Mn} / \mathrm{Sb}$ and $\mathrm{Mn} / \mathrm{Ni}$ intensity ratios bend upward with increasing emission angle and annealing time, whilst the $\mathrm{Ni} / \mathrm{Sb}$ ratio is not only very small compared with the other two, but also almost constant as a function of emission angle. This result can be explained only if the surface region (high emission angles) is $\mathrm{Mn}$ rich and so, the proportionality of the three elements is altered as we probe closer to the surface terminal layer.

In order to analyze the Mn surface segregation phenomenon quantitatively, we again used the fitting procedure introduced above. The measured XPS ratios are fitted with a model in which the Mn atoms can segregate to both vacancy sites as well as replace both $\mathrm{Sb}$ and $\mathrm{Ni}$ atoms. The $\mathrm{Mn}$ atomic fraction in the surface region can be written as:

$$
f_{j}(\mathrm{Mn})=b+\delta \exp \left(-\frac{\mathrm{j} d}{G}\right)
$$

where $b$ is the bulk atomic fraction of 0.5 for the $\mathrm{Mn} / \mathrm{Sb}$ layers $(j=$ even) and $b=0$ for the $\mathrm{Ni} /$ vacancies planes. The contribution to the intensity peaks for each elemental core can be summed for the corresponding layer, as follows:

$$
\begin{aligned}
& I(\mathrm{Mn})=\sum_{j=0}^{\infty} 0.5 \exp \left(-\frac{2 \mathrm{j}}{\lambda_{\mathrm{Mn}} \cos \theta}\right)+\sum_{j=0}^{\infty} \delta \exp \left(-\frac{\mathrm{j}}{G}-\frac{\mathrm{j}}{\lambda_{\mathrm{Mn}} \cos \theta}\right) \\
& =\frac{0.5}{1-\exp \left(-\frac{2}{\lambda_{\mathrm{Mn}} \cos \theta}\right)}+\frac{\delta}{1-\exp \left(-\frac{1}{G}-\frac{1}{\lambda_{\mathrm{Mn}} \cos \theta}\right)} \\
& I(\mathrm{Ni})=\sum_{j=0}^{\infty} 0.5 \exp \left(-\frac{2 \mathrm{j}+1}{\lambda_{\mathrm{Ni}} \cos \theta}\right)-\sum_{j=0}^{\infty} \delta \exp \left(-\frac{2 \mathrm{j}+1}{G}-\frac{2 \mathrm{j}+1}{\lambda_{\mathrm{Ni}} \cos \theta}\right) \\
& =\frac{0.5 \exp \left(-\frac{1}{\lambda_{\mathrm{Ni}_{\mathrm{i}} \cos \theta}}\right)}{1-\exp \left(-\frac{2}{\lambda_{\mathrm{N}_{\mathrm{i}} \cos \theta}}\right)}-\frac{\delta \exp \left(-\frac{1}{G}-\frac{1}{\lambda_{\mathrm{Ni}} \cos \theta}\right)}{1-\exp \left(-\frac{2}{G}-\frac{2}{\lambda_{\mathrm{N}_{\mathrm{i}} \cos \theta}}\right)} \\
& I(\mathrm{Sb})=\sum_{j=0}^{\infty} 0.5 \exp \left(-\frac{2 \mathrm{j}}{\lambda_{\mathrm{Sb}} \cos \theta}\right)-\sum_{j=0}^{\infty} \delta \exp \left(-\frac{2 \mathrm{j}}{G}-\frac{2 \mathrm{j}}{\lambda_{\mathrm{Sb}} \cos \theta}\right) \\
& =\frac{0.5}{1-\exp \left(-\frac{2}{\lambda_{\mathrm{Sh}} \cos \theta}\right)}-\frac{\delta}{1-\exp \left(-\frac{2}{G}-\frac{2}{\lambda_{\mathrm{Sb}} \cos \theta}\right)}
\end{aligned}
$$

where the $\lambda$ s are the corresponding mean free paths given in table 4 , and the characteristic segregation parameters $\delta$ and $G$ were considered fitting parameters. The fitting proce- 


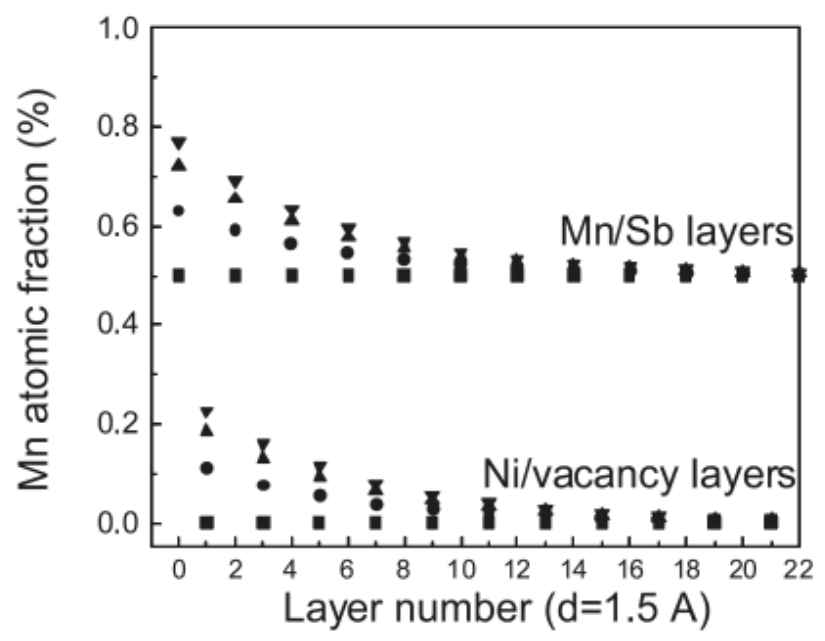

Figure 21. The Mn concentration in the near surface region obtained from fits of the angle dependent XPS ratios. The symbols are identical to the ones used in figure 20. Adapted from [48].

Table 5. The fitting parameters used for modeling the experimental ratios in figure 20. $\delta$ represents the segregation at the surface-vacuum interface and $G$ is the segregation depth, expressed both in $\AA$ and in units of distance $(d=1.5 \AA)$ between layers.

\begin{tabular}{llll}
\hline Annealing time & $G(d=1.5 \AA)$ & $G(\AA)$ & $\delta(\%)$ \\
\hline 5 min at $400^{\circ} \mathrm{C}$ & 5.7 & 8.55 & 13 \\
15 min at $400^{\circ} \mathrm{C}$ & 5.7 & 8.55 & 22 \\
30 min at $400^{\circ} \mathrm{C}$ & 5.7 & 8.55 & 27 \\
\hline
\end{tabular}

dure was: (1) assume a certain stacking sequence, (2) fit the $\mathrm{Mn} / \mathrm{Ni}$ experimental ratio by adjusting $\delta$ and $G$, (3) calculate the $\mathrm{Mn} / \mathrm{Sb}$ ratio given the fit to the $\mathrm{Mn} / \mathrm{Ni}$ ratio, and (4) if the calculated ratios of the previous steps do not fit the corresponding experimental ones, then try a new stacking sequence. The best results of the fitting procedure for three different surfaces are shown as continuous lines in figure 20, together with stoichiometric surface (assumed to be segregation free). The fitting parameters used in the models are presented in table 5 .

The surface concentration profiles were easily constructed using equation (8) and the values of $\delta$ and $G$ obtained above. Figure 21 presents the Mn concentration dependence upon layer number for the three heavily annealed surfaces. Note that the same figure also contains the constant $\mathrm{Mn}$ atomic fraction of 0.5 for the $\mathrm{Mn} / \mathrm{Sb}$ layers and 0 for the $\mathrm{Ni} /$ vacancy layers, characteristic of a stoichiometric structure for the freshly prepared surface. This surface termination of $\mathrm{MnSb}$, for the stoichiometric surface is entirely consistent with the predictions of Jenkins and co-workers $[38,43,75,76]$ and others $[42,44]$.

When equilibrium is established between the surface and the bulk, the surface enthalpy is quite different from the bulk [65]. Surface segregation is a strong indication that the surface enthalpy differs significantly from the bulk, in the context of standard statistical models. In a simple statistical mechanical model of segregation [83], the total free energy, $F$, for the system is written as:

$$
F=\sum_{i} n_{i}^{\mathrm{b}} g_{i}^{\mathrm{b}}+n_{i}^{\mathrm{s}} g_{i}^{\mathrm{s}}-k_{\mathrm{B}} T \ln \Omega,
$$

where $n_{i}^{\mathrm{b}}$ and $n_{i}^{\mathrm{s}}$ are the number of the bulk and surface atoms of type $i$, with individual 


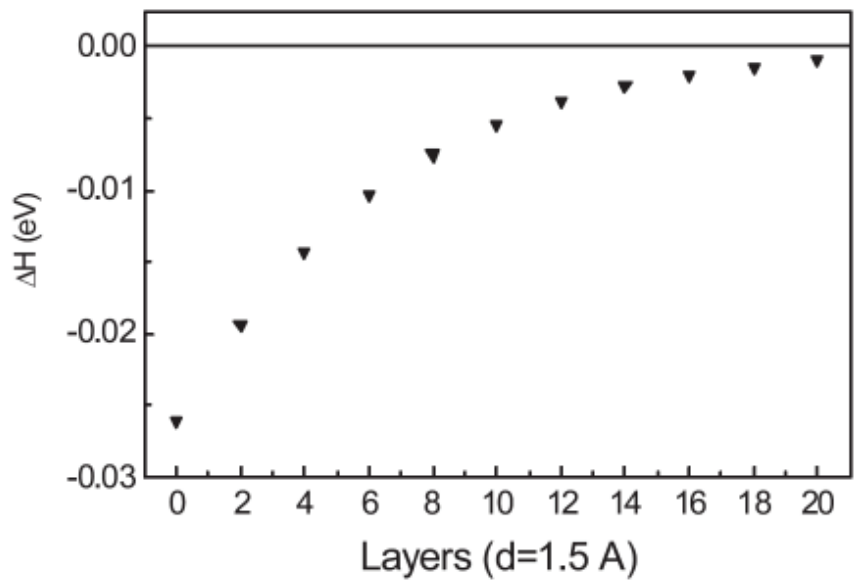

Figure 22. The enthalpy difference between the surface and the bulk based on the most extensive Mn segregation shown in Figure 21 (down-triangles). Adapted from [48].

free energies $g^{\mathrm{b}}$ and $g_{i}^{\mathrm{s}}$, respectively; $k_{\mathrm{B}}$ is the Boltzmann constant, $T$ is the temperature of the system, and $\Omega$ is the entropy due to the mixing of the components. For a two-component system, an Arrhenius expression can be written as:

$$
\frac{n_{\mathrm{A}}^{\mathrm{s}}}{n_{\mathrm{B}}^{\mathrm{s}}}=\frac{n_{\mathrm{A}}^{\mathrm{b}}}{n_{\mathrm{B}}^{\mathrm{b}}} \exp \left(-\frac{H}{k_{\mathrm{B}} T}\right),
$$

where $H$ is the enthalpy of the segregation. Equation (13) implies that the surface segregation is a competition to minimize the total free energy by a maximization of entropy by evenly mixing the two elements. Using the bulk concentration values for the A and B components, one can easily extract the dependence of the segregation enthalpy of the atomic fraction.

We can calculate the dependence of enthalpy as a function of layer number, as demonstrated in figure 22, for the most extensively Mn segregated surface (after $30 \mathrm{~min}$ annealing at $400{ }^{\circ} \mathrm{C}$ ). This difference in enthalpy can be related to the creation of a surface electronic structure very different from the bulk (i.e. surface states for the stoichiometric surface), can result in a new surface lattice structure distinct from the bulk and, of course, can become the driving force for segregation. This energy difference, between the surface and the bulk, appears to be more than sufficient to overcome the energy barriers to defect formation, that have been thought to hinder defect mediated reduction in polarization [84, 85]. In addition, defects and variations in the local lattice order are believed to strongly influence the net electron polarization near the Fermi level, as discussed in the next section.

\section{Surface and bulk Debye temperatures}

The thermal motion of the surface atoms dictates many of the physical and chemical properties such as: surface diffusion [86, 87]; anharmonicity leading to roughening transitions [88-90]; surface reconstructions [91, 92]; which all influence the extent of compositional inhomogeneities, including defects. The key descriptive parameter of the dynamic motions of atoms on the surface, as well as in the bulk, is the Debye temperature.

We investigated the effective surface Debye temperature of the NiMnSb(100) using photoemission (XPS) and low energy electron diffraction (LEED) techniques which we compared to the bulk Debye temperature calculated from the phonon dispersion curves 
obtained from inelastic neutron scattering [93]. This comparison is one of a very limited number of such studies in complex compound materials $[94,95]$ and the first for the Heusler alloys.

The dynamic motions normal to the film surface dominate the surface Debye temperature $\theta_{\mathrm{D}}$ determined by most electron spectroscopy techniques using the Debye-Waller model $[96,97]$. This surface $\theta_{\mathrm{D}}$ is dominated by the atoms' dynamic motions parallel to the scattering vector and typically does not contain significant in-plane or anharmonic contributions to the true surface Debye temperature. We regard this parameter $\left(\theta_{\mathrm{D}}\right)$ as the measure of the dynamic motions of specific atoms along the direction of the surface normal. For LEED spot with intensity $I$, we used the formula:

$$
I-I_{0} \exp [-2 W(T)]
$$

where $W(T)$ is the Debye-Waller factor. $W$ is given by:

$$
2 W(T)=|\Delta k|^{2}\left\langle u_{0}\right\rangle^{2},
$$

in which $k$ is the wavevector transfer and $u_{0}$ is the mean squared displacement of the atoms. In the framework of Debye theory, the factor $W$ can be approximated in the first order as $[96,98]$ :

$$
2 W=\frac{3 \hbar^{2}(\Delta k)^{2} T}{m k_{\mathrm{B}} \theta_{\mathrm{D}}^{2}},
$$

where $W$ is the Debye-Waller factor, $\hbar$ is Planck constant, $T$ is the sample temperature, $\hbar(k)$ is the electron momentum transfer, $m$ is the mass of the of the scattering centre, $k_{\mathrm{B}}$ is the Boltzmann constant, and $\theta_{\mathrm{D}}$ is the effective surface Debye temperature.

The scattering vector is obtained from equation:

$$
\Delta k=2 k \cos \frac{\theta}{2}=\frac{4 \pi}{\lambda} \cos \frac{\theta}{2},
$$

where $\theta$ is the angle between the incoming and outgoing electron beams and $\lambda$ is the wavelength of the electrons. The mass of the scattering center is approximated by an average value of the three different atomic masses contained in a unit cell:

$$
m=\left[M_{\mathrm{Mn}}+M_{\mathrm{Ni}}+M_{\mathrm{Sb}}\right] / 3=78.45 \mathrm{au} .
$$

Figure 23 shows the temperature dependence of the intensities from backscattered low energy electron diffraction, after background subtraction $\left(I_{\mathrm{bk}}\right)$ and normalization to the value $\left(I_{0}\right)$ at the lowest temperature. The three sets of data shown were taken using different incident electron energies of 33, 58 and $83 \mathrm{eV}$. The angle $\theta$ from equation (15) varied very little in our experiment and the change in scattering vector $(\Delta k)$ largely comes from the different electron energies employed. The change in LEED intensities, plotted in figure 23 as the slope of $\ln \left[\left(I-I_{\mathrm{bk}}\right) / I_{0}\right]$, increased with increasing $(\Delta k)$ or with increasing incident (and backscattered) electron energies. Using equation (14), the values obtained for the effective surface Debye temperature are $140 \pm 20 \mathrm{~K}$ (at $33 \mathrm{eV}$ incident electron energy), 143 $\pm 20 \mathrm{~K}$ (at $58 \mathrm{eV}$ ) and $154 \pm 20 \mathrm{~K}$ (at $83 \mathrm{eV}$ ). The electron's mean free path increases and so does the probing depth with increasing incident (and backscattered) electron energies. We consider that the effective Debye temperature obtained at the lowest electron energies is the most representative of the dynamic normal atomic motions in the thin film surface region. The other two values, at higher electron energies, include the normal vibrations of the atoms situated increasingly deeper in the selvedge (near surface) region.

In the case of photoemission spectroscopy (XPS), the most common geometry used is the one in which the outgoing electrons are emitted in the normal direction $(\theta=0)$. In this case the momentum transfer is equal to the momentum of the emitted electron: $\Delta k=$ 


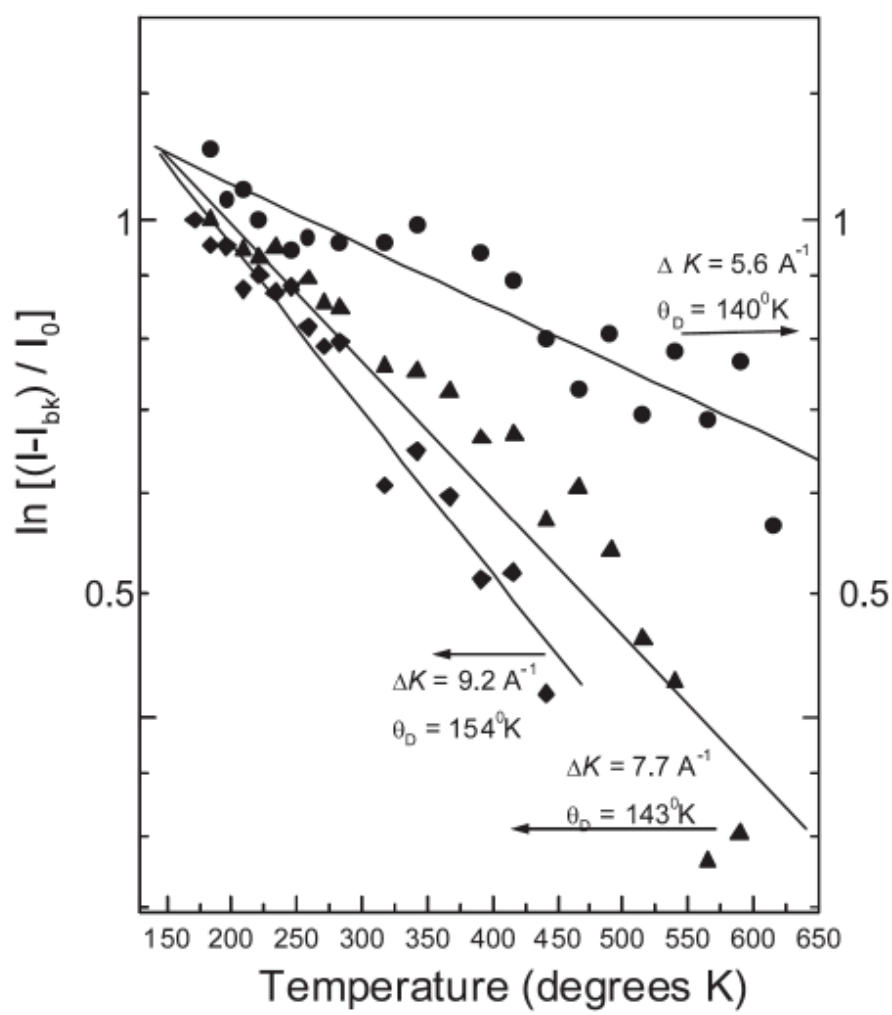

Figure 23. Logarithm of the intensities of the diffracted electron spots obtained in LEED, after background subtraction $\left(I_{\mathrm{bk}}\right)$ and normalization to the value $\left(I_{0}\right)$ at the lowest temperature. The three sets of data were obtained using different incident electron energies: $33 \mathrm{eV}$ (circles), $58 \mathrm{eV}$ (up-triangles) and $83 \mathrm{eV}$ (diamonds). The direction of the incident electrons was along the surface normal. Adapted from [93].

$2 \pi / \lambda$. In our experiments, we monitored the photoelectron intensity from the $3 \mathrm{~d}$ shell of the $\mathrm{Sb}$ atoms using $\mathrm{MgK} \alpha$ radiation $(1253.6 \mathrm{eV}$, with a FWHM of $0.65 \mathrm{eV}$ ), and appropriately corrected for the scattering centre mass. The temperature dependence of the $3 \mathrm{~d}_{3 / 2}$ core level of antimony, obtained from x-ray core level photoemission, is shown in figure 24. This particular core level has a narrow and intense line shape at all temperatures. The temperature dependence of the $\mathrm{Sb} 3 \mathrm{~d}_{3 / 2}$ core level peak intensities is shown in the right panel of figure 24 and using equations (12) and (14) we obtain a value of $144 \pm 10 \mathrm{~K}$ for the effective surface Debye temperature. The mean free path of the ejected electrons in normal emission is no more than $20 \AA$ from the free surface and so the sample probing depth is limited to the surface and selvedge region.

For comparison with the above values, we extracted the Debye temperature for bulk single crystals from the wavevector dependent inelastic neutron scattering (a bulk sensitive technique) [90]. Neutron inelastic scattering is a common technique for mapping the dispersion curves for acoustic and optical phonons, as well as the magnon dispersion. For values of the scattering vector $q$ close to zero, the dispersion of the acoustic oscillatory modes is linear, and the slope of this linear dependence is related to specific elastic constants, depending on the scanning direction.

The bulk Debye temperature of single crystals can be estimated from application of the Debye model and the phonon dispersion curves [99]. In this model, the upper limit $v_{\mathrm{D}}$ 

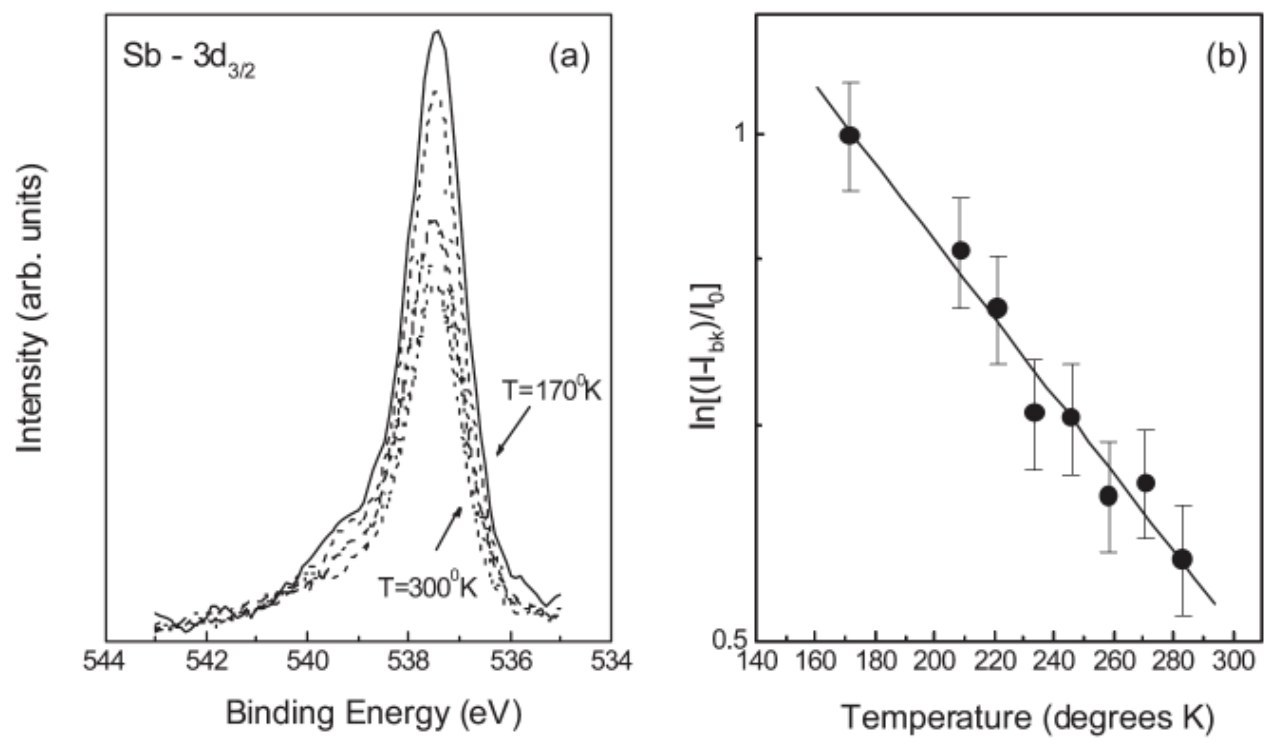

Figure 24. (a) Normal x-ray photoemission spectra of $\mathrm{Sb} 3 \mathrm{~d}_{3 / 2}$ core level for various tem-

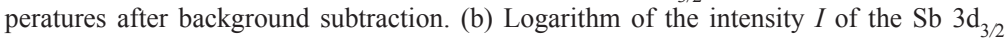
core level versus temperature after background $\left(I_{\mathrm{bk}}\right)$ subtraction and normalization to the value $I_{0}$ at the lowest temperature. Adapted from [93].

to the frequency is obtained from the normalizing condition that the total number of oscillatory modes is equal to $3 r N_{\mathrm{A}}$ per mole, where $r$ is the number of atoms per unit cell (and $N_{\mathrm{A}}$ is Avogadro's number). The quantity $v_{\mathrm{D}}$ is related to the parameter $\theta_{\mathrm{D}}$ by:

$$
v_{\mathrm{D}}=\frac{k_{\mathrm{B}}}{h} \theta_{\mathrm{D}} \text {. }
$$

Thus, the frequency limit $v_{\mathrm{D}}$ and the related parameter $\theta_{\mathrm{D}}$, the Debye temperature, can be obtained from the formula [100]:

$$
\frac{4 \pi V}{3}\left(c_{\mathrm{L}}^{-3}+2 c_{\mathrm{T}}^{-3}\right) \nu_{\mathrm{D}}^{3}=3 r N_{\mathrm{A}},
$$

in which $V$ is the molar volume and $c_{\mathrm{L}}, c_{\mathrm{T}}$ are the corresponding longitudinal and transverse wave velocities. For cubic crystals, the velocities are related to the elastic constants $C_{11}$ and $C_{44}$ as follows [100]:

$$
\begin{gathered}
c_{\mathrm{L}}=\sqrt{\frac{C_{11}}{\rho}} \\
c_{\mathrm{T}}=\sqrt{\frac{C_{44}}{\rho},}
\end{gathered}
$$

where $\rho=7610 \mathrm{~kg} \mathrm{~m}^{-3}$ is the mass density. (Note that the elastic constants ' $C$ ' have the dimensions of [energy]/[volume] [100].)

The inelastic neutron scattering experiments [101] were carried out at the Institute Laue-Langevin, using the IN1 (hot neutrons) and IN8 (thermal neutrons) spectrometers, in order to cover all transfer energies values from 0 to $120 \mathrm{meV}$. The measurements were made on three single crystals, with sizes ranging from 100 to $2000 \mathrm{~mm}^{3}$ whose mosaic 


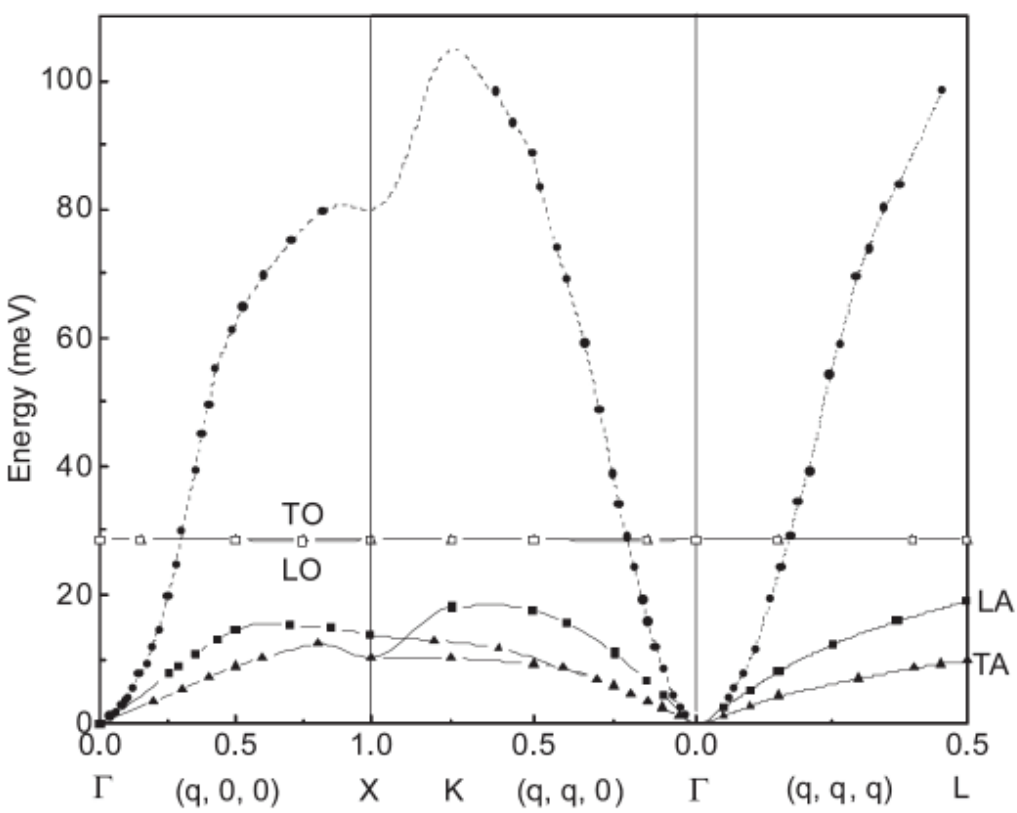

Figure 25. Dispersion curves for magnons (circles), longitudinal phonons (squares), and transverse phonons (up-triangles) at $300 \mathrm{~K}$ obtained in neutron scattering. No magnetic field was applied. Adapted from [93, 101].

spread was less than $0.5^{\circ}$. Excitations were investigated mainly at room temperature in the direction of the high symmetry axis (100), (110) and (111). No magnetic field was applied. Constant energy scans were generally performed to investigate the magnon dispersion, whereas constant- $q$ scans were used to locate and map the wavevector dependence of the acoustic and optical phonons. Figure 25 presents the complete experimental phonons and magnons dispersion curves at $300 \mathrm{~K}[51,93,101]$.

For a material with three atoms in the primitive cell, we generally expect three acoustic and six optical branches, whereas only one (twofold-degenerate) branch of magnons was observed. Longitudinal acoustic phonons reach a maximum energy for $q$ close to $(0.6$, $0,0)$ in the $\langle 100\rangle$ direction and $(0.7,0.7,0)$ in the $\langle 100\rangle$ direction. One very flat optical branch was observed at energy of about $29 \mathrm{meV}$, with little difference in energy for longitudinal and transverse directions. (One explanation for this dispersionless optical mode is the arrangement of the $\mathrm{Ni}$ atoms in the cubic structure. Among the closest neighbor lattice sites to nickel are the vacancy sites.)

The elastic constants can be easily extracted from the slope of the acoustic modes in each $\Gamma$ point, depending on the scanning direction. For the $\langle 100\rangle$ longitudinal mode, $C_{11}$ $=138 \mathrm{GPa}$, for the $\langle 100\rangle$ transverse mode $1, C_{44}=45 \mathrm{GPa}$ and for the $\langle 100\rangle$ transverse mode $2, C_{12}=48 \mathrm{GPa}[93,101]$. Using these values to calculate the longitudinal and transverse mode velocities in the equation (21), we obtained a value of the bulk Debye temperature of $312 \pm 5 \mathrm{~K}$ [93].

Almost independently of surface orientation we would expect that the effective surface Debye temperature is of the order of $70 \%$ (i.e. $1 / \sqrt{2}$ ) of the bulk value of the Debye temperature [96-98]. This is due to the fact that the surface atoms have only half of the number of nearest neighbors that surround the bulk atoms [96]. Comparing the values of the Debye temperature obtained for the NiMnSb(100) Heusler alloy, the surface tempera- 
ture is almost $45 \%$ of the bulk value. This drop in the Debye temperature between the surface and the bulk is not unexpected, under our preparation conditions, if we take into account that the surface and selvedge regions have different compositions from the bulk [32, 48]. Indeed, as shown in [48], manganese segregation occurs at the NiMnSb(100) surface and, in the surface region, the vacancies in the nickel plane(s) are filled with Mn while the $\mathrm{Ni}$ to $\mathrm{Sb}$ ratio remains relatively unperturbed. In addition, there are several other phenomena that may contribute to the lowering of the surface Debye temperature with respect to the bulk. The motion of the surface atoms is not isotropic, the force constants at the surface can be appreciably different from those in the bulk, and, in general, the surface may contain anharmonic phonon modes that differ from the bulk $[102,103]$.

The reduced surface Debye temperature will lower the barriers to surface segregation and can be a consequence of the compositional differences due to segregation. The polarization asymmetry representative of the vacuum-alloy interface could be strongly influenced by the thermal oscillations of the atoms in the surface region [41].

\section{Characterization of the surface electronic structure}

The understanding of the electronic structure is the key to gathering insight into many characteristics of a solid, including the dielectric, magnetic and bonding properties. Fundamental issues related to magnetism, such as the exchange coupling, the spin-orbit coupling, the magnetic moment, and the Curie temperature are determined by the electronic structure. The valence and conduction band structure can be experimentally investigated with photoemission, inverse photoemission and x-ray absorption.

Using the surface preparation technique described in section 4, we were able to characterize an epitaxial and stoichiometric surface of $\mathrm{NiMnSb}$. Establishing the correct stoichiometric surface composition of the $\mathrm{NiMnSb}(100)$ surface, determined with both LEED and XPS as demonstrated in section 4, was essential for the spin polarized IPES, which is a highly surface sensitive technique. The choice of spin polarized inverse photoemission for determining polarization is dictated by several considerations. Spin polarized inverse photoemission can be more readily compared to magnetic circular dichroism and spin polarized appearance potential spectroscopy as all three probe the unoccupied band structure [104], while spin polarized photoemission does not. Furthermore, depolarization due to the ground state non-quasiparticle density of state should be most significant in the unoccupied density of states just above the Fermi level for NiMnSb [61].

The NiMnSb sample was installed in a UHV system, inside a coil which is used to switch the in-plane magnetization direction. A pulsed 400 Oe magnetic field was applied along the $\langle 110\rangle$ easy magnetization direction, representing a far larger value than the sample's saturation and coercive fields of 40 and 32 Oe, respectively. The spin up and down densities of states near the Fermi level for a stoichiometric surface (figure 26) were measured at room temperature and normal incidence of the incoming electron beam. The polarization at the Fermi level is as high as $67 \pm 9 \%$, or nearly $100 \%$ above background, as shown in the lower panel of figure 26 . Note that the spin asymmetry shown in figure 26 is calculated as a function of incident electron energy using equation:

$$
P=\frac{N_{\uparrow}-N_{\downarrow}}{N_{\uparrow}+N_{\downarrow}},
$$

where $N_{\uparrow}$ and $N_{\downarrow}$ are the spin up (down) density of states, respectively. This asymmetry value measured in spin polarized IPES is significantly higher than the polarization asymmetry of $50 \%$, at room temperature, measured from a polycrystalline sample using spin 


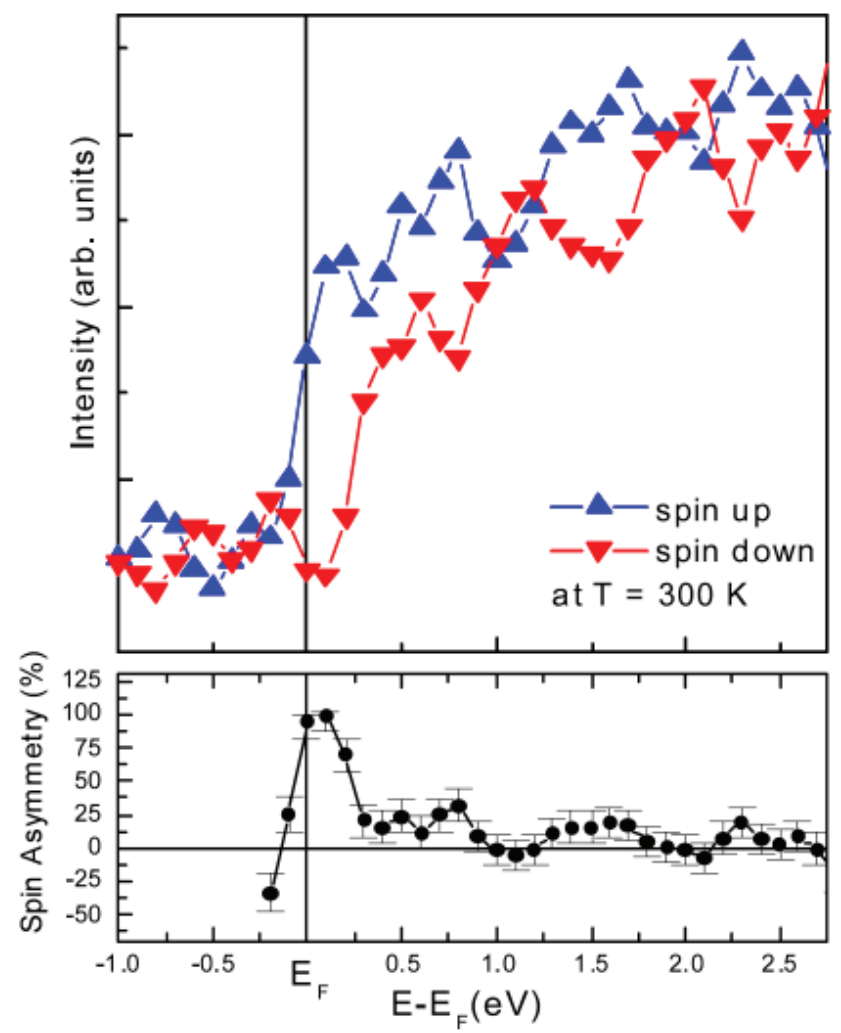

Figure 26. Spin polarized unoccupied electronic bands from spin polarized inverse photoemission spectroscopy at normal incidence for the stoichiometric NiMnSb surface, at $300 \mathrm{~K}$. The bottom panel shows the corresponding spin asymmetry near $E_{\mathrm{F}}$. Adapted from [32].

polarized photoemission [26], even when the background is included, and much higher that the spin polarized inverse photoemission data obtained by Grenz [30]. For these previously reported spin polarized photoemission measurements, Sb segregation was observed, which would tend to lower the polarization. The data taken at $300 \mathrm{~K}$ from the stoichiometrically ordered alloy surface indicate a conduction band edge, in spin minority, at about $250 \mathrm{meV}$ above the Fermi level. The high values of polarization near the Fermi level in our spin polarized inverse photoemission data at room temperature do suggest that depolarization (a reduction of polarization from 100\%), due to the ground state nonquasiparticle density of states [61, 62], is quite small.

According to calculations for NiMnSb [2], the Fermi level intersects the centre of the gap (or slightly above centre), in spin minority. Thus, these spin polarized data (at the surface Brillouin zone centre or $k_{\|}=0$ ) are consistent with both a calculated gap in spin minority of about $0.5 \mathrm{eV}$ and a half-metallic system. The data may not, however, be considered as conclusive proof that NiMnSb is indeed a half-metallic system. Such a definitive statement would require mapping the electronic densities of states in more than one $k$ space points across the Brillouin zone, particularly in the vicinity of the spin majority Fermi level crossings as well as ensuring that the polarization is not a result of surface states. The short "lifetime" of the clean, stoichiometric surfaces (of the order of 4-5 h) made it impossible to perform the required studies needed to prove the half-metallic character across the entire Brillouin zone, as the final spectrum must be obtained by 


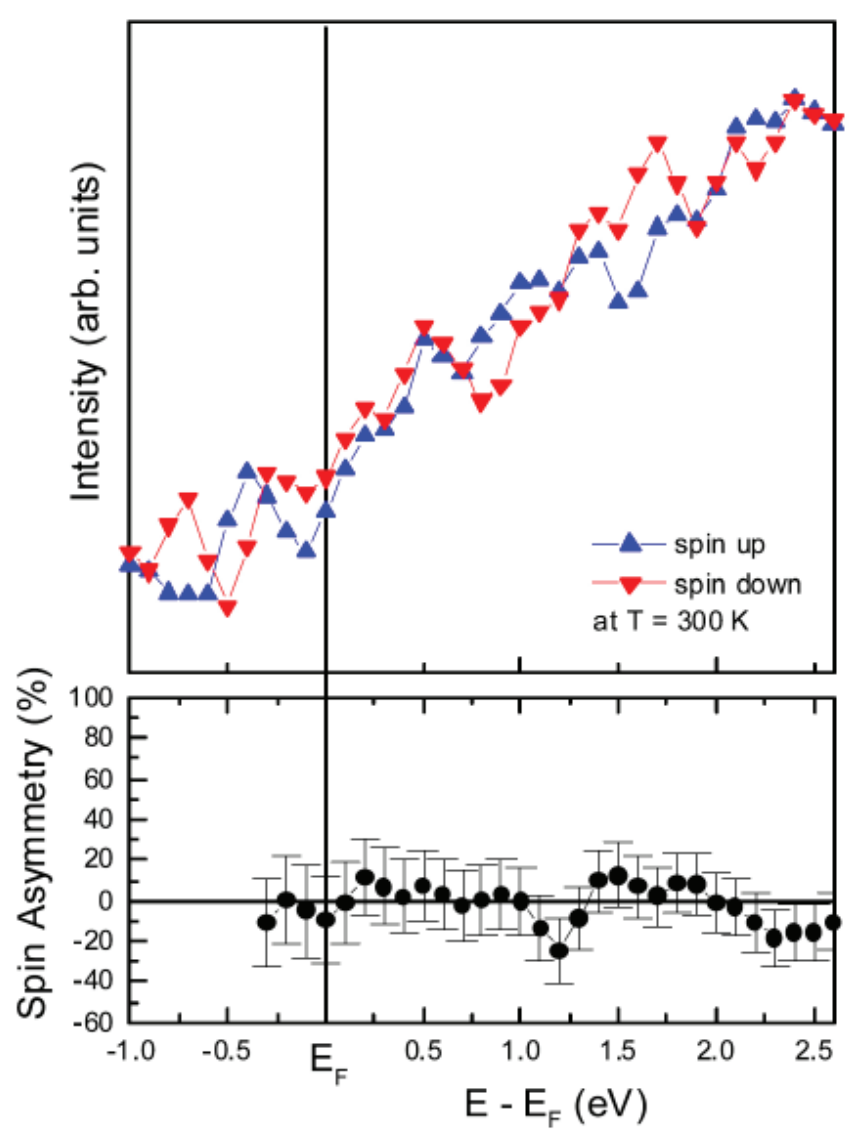

Figure 27. Spin polarized unoccupied electronic bands from spin polarized inverse photoemission spectroscopy at normal incidence for the $\mathrm{Mn}$ rich $\mathrm{NiMnSb}$ surface, at $300 \mathrm{~K}$. The bottom panel shows the corresponding spin asymmetry near $E_{\mathrm{F}}$. Adapted from [32].

summing experiments from several samples stoichiometric surface. Sputtering and annealing of $\mathrm{NiMnSb}$ ordered surfaces (performed to ensure contamination free surfaces) leads to a degradation of surface composition, including Mn segregation, as presented in section 4 , and such data cannot be used. What experimental band mappings that do now exist have been taken using photoemission (where the data collection is faster) are spin integrated [105].

The influence of the Mn segregation on the unoccupied band structure of NiMnSb results in a drop of the polarization asymmetry and a loss of density of states near the Fermi level, as can be observed in figure 27. These changes in polarization and density of states represent a decrease in surface metallicity along with the disappearance of the inferred gap in the spin minority subband and, therefore, loss of half-metallic character in the surface $[11,49,50]$. The behavior of this segregated surface is more reminiscent of a $\mathrm{MnSb}$ binary alloy [106-109] or of an antiferromagnetically aligned Mn rich surface layer, than of the expected electronic structure of NiMnSb.

The unoccupied bands above the Fermi level obtained from IPES, can be assigned to each atomic constituent in the sample by comparing the IPES and XAS spectra [104]. The sample used in the XAS study was capped with $25 \AA$ of $\mathrm{Al}$ for protection against oxida- 


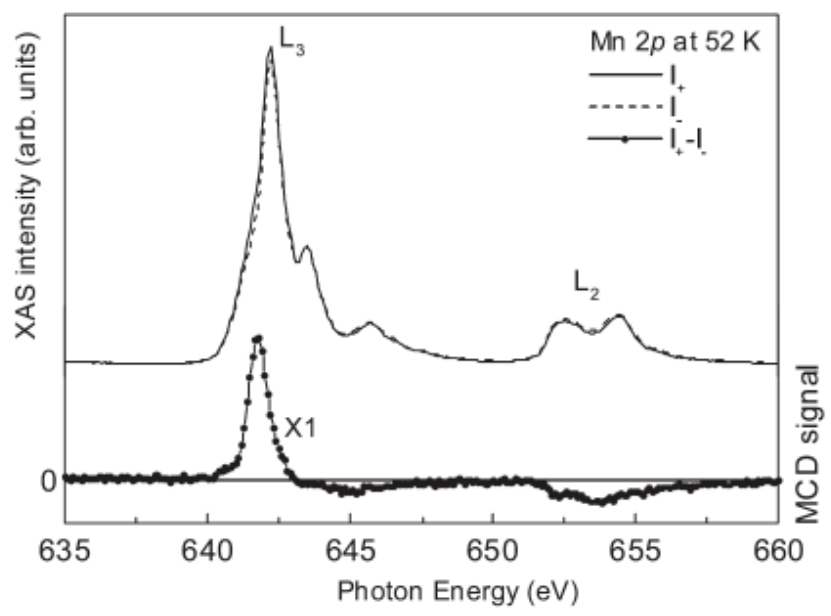

Figure 28. Absorption spectra for $\mathrm{L}_{3}$ and $\mathrm{L}_{2}$ edges of $\mathrm{Mn}$ at $52 \mathrm{~K}$. The lower curve shows the corresponding MCD signal obtained as the difference between XAS signals with opposite magnetization directions. Adapted from [33].

tion as is well known to have a very small cross section for the energies involved in this technique. Given that the total Al thickness was too small to affect significantly the probing depth of the absorption spectra, we assume that $\mathrm{Al}$ does not alter the NiMnSb surface composition and, therefore, the XAS spectra must be compared to the IPES spectra measured on a clean, stoichiometric surface, free of Mn segregation.

The XAS and XMCD spectra were measured at the U4B beamline located at the National Synchrotron Light Source (NSLS) at Brookhaven National Laboratory. The XAS spectra were measured by total electron yield detection (TEY) in $45^{\circ}$ sample orientation with respect to the incoming beam, in vacuum less than $5 \times 10^{-10}$ Torr. We recorded two total electron yield spectra, one with the incident light helicity (circular polarization) oriented parallel to the remanent magnetization of the sample, and one antiparallel. The XAS spectrum is the average of these two spectra, while the XMCD is the difference between the two. For the measurements included in this work, the light helicity was kept constant while the remanent magnetization of the sample was reversed by reversing a magnetic field applied parallel to the sample. The spectra were normalized to the incident flux via the current measured through a gold mesh that was placed in the beam in front of the sample.

In accordance with the corresponding selection rules detailed in [104], we have measured the absorption edge induced by the transition from the $2 p$ initial state to the $3 \mathrm{~d}$ final state, for both Mn and Ni ferromagnetically aligned sublattices. The XAS and MCD spectra obtained for the $\mathrm{Mn} \mathrm{L}_{2}$ and $\mathrm{L}_{3}$ edges at $50 \mathrm{~K}$ are shown in figure 28 . The spinorbit splitting for the $\mathrm{Mn}$ atoms is given by the energy difference between the $\mathrm{L}_{2}$ and $\mathrm{L}_{3}$ peaks, approximately $11.5 \mathrm{eV}$. This value is very close to the spin-orbit coupling found in the Mn single crystal. The direct effect of the crystalline lattice on Mn atoms can be observed in the fine structure of both edges. The $\mathrm{L}_{3}$ peak is split in three, while the $\mathrm{L}_{2}$ edge is a doublet. The calculations found in [110] correspond well with our data in the case of cubic symmetry of the crystalline Mn lattice, which also represents the symmetry of the half-Heusler alloy. The splitting introduced by the crystal field is stronger than any multiplet effects ( $2 p-3 d$ hybridization), which are shown to influence the L edge of any transitional metal [111]. The Mn MCD signal, shown in the lower part of each panel in figure 28 , is positive in the $L_{3}$ edge and negative for the $L_{2}$ edge. 


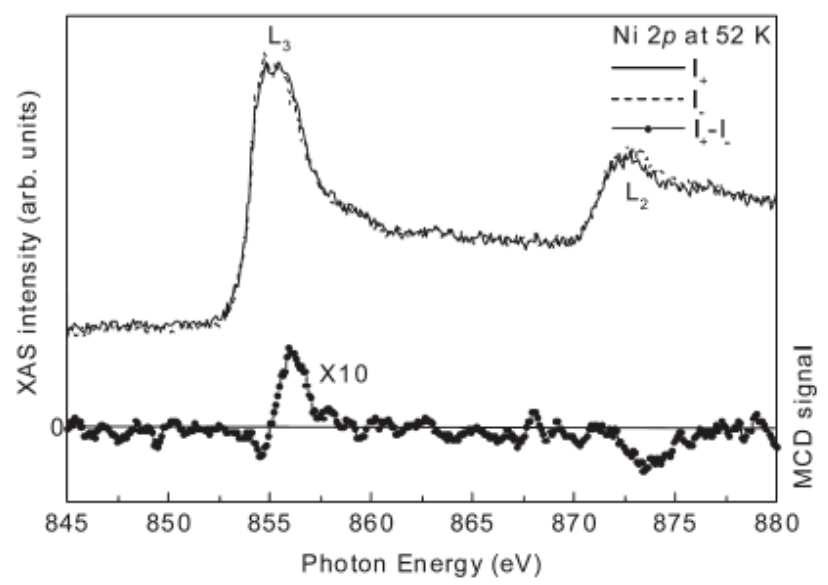

Figure 29. Absorption spectra for $\mathrm{L}_{3}$ and $\mathrm{L}_{2}$ edges of $\mathrm{Ni}$ at $52 \mathrm{~K}$. The lower curve shows the corresponding MCD signal obtained as the difference between XAS signals with opposite magnetization directions. The MCD intensity scale is reduced by a factor of 10 compared to the MCD intensity scale of Mn in figure 28. Adapted from [33].

The corresponding Ni XAS and MCD spectra are presented in figure 29 at a temperature of $52 \mathrm{~K}$. The spin-orbit coupling strength is $16.8 \mathrm{eV}$ - the same as in a Ni single crystal. The fine structure of the Ni absorption peaks is not very pronounced, consistent with a $3 \mathrm{~d}^{9}$ initial state [110]. The crystal field has no influence on the shape of the L edge corresponding to an almost full $3 \mathrm{~d}$ band metal [111]. The Ni dichroic signal remains positive (negative) for the $\mathrm{L}_{3}\left(\mathrm{~L}_{2}\right)$ edge as in the case of $\mathrm{Mn}$, indicating a ferromagnetic coupling between $\mathrm{Mn}$ and Ni. Note that the intensity of the Ni dichroic signal is ten times less than the Mn dichroic signal, as shown in the figure 29. This drop of the dichroic intensity is in agreement with the experimental values obtained for the local moments using polarized neutron diffraction $[33,59]$. The $\mathrm{Sb} 3 \mathrm{~d}$ XAS spectrum (not shown here) is dominated mainly by the oxygen peak from the $\mathrm{Al}$ cap layer, which interferes with the $3 \mathrm{~d}$ spin-orbit coupling. The presence of oxygen (for the oxidation of the aluminum capping layer) introduces great difficulties in interpreting the shape of the Sb XAS spectrum but this should not affect the MCD signal. The local magnetic moment of Sb, given by the total area of the MCD signal, is negligible - of the order of $0.1 \mu_{\mathrm{B}}$ and in agreement with band calculations [31].

The comparison between spin polarized inverse spectrum of the unoccupied density of states and x-ray absorption spectra (XAS) for Mn $2 p$ and for Ni $2 p$ can be made, due to the fact that both techniques generally probe the same final states, as noted above [104]. Figure 30 shows the comparison of the SPIPES and XAS spectra for NiMnSb(100) at room temperature. For both MCD and XAS spectra, the conduction band edge (the approximate Fermi energy) for the XAS spectra is assigned on the basis of the experimentally measured core level binding energies for $\mathrm{NiMnSb}(100)$ : $640.2 \mathrm{eV}$ for the $\mathrm{Mn} 2 \mathrm{p}_{3 / 2}$ and $853.3 \mathrm{eV}$ for the $\mathrm{Ni} 2 \mathrm{p}_{3 / 2}$ (figure 20). In the XAS spectra, the unoccupied states are shifted to slightly higher energies above $E_{\mathrm{F}}$ than in SPIPES, as expected from the perturbation of the Coulomb interaction with the photoexcited core-hole. Nonetheless, from this comparison, we can establish a rough correspondence of the unoccupied states in inverse photoemission. The density of states just above $E_{\mathrm{F}}$, at approximately $0.63 \mathrm{eV}$, is largely $\mathrm{Mn}$ in origin (figure 30), as derived from our XAS/MCD studies, and this is generally consistent with other XAS/MCD studies $[112,113]$, and recent spin polarized appearance spectroscopy measurements [31]. Our Mn XAS spectra are characteristic of a Mn d ini- 


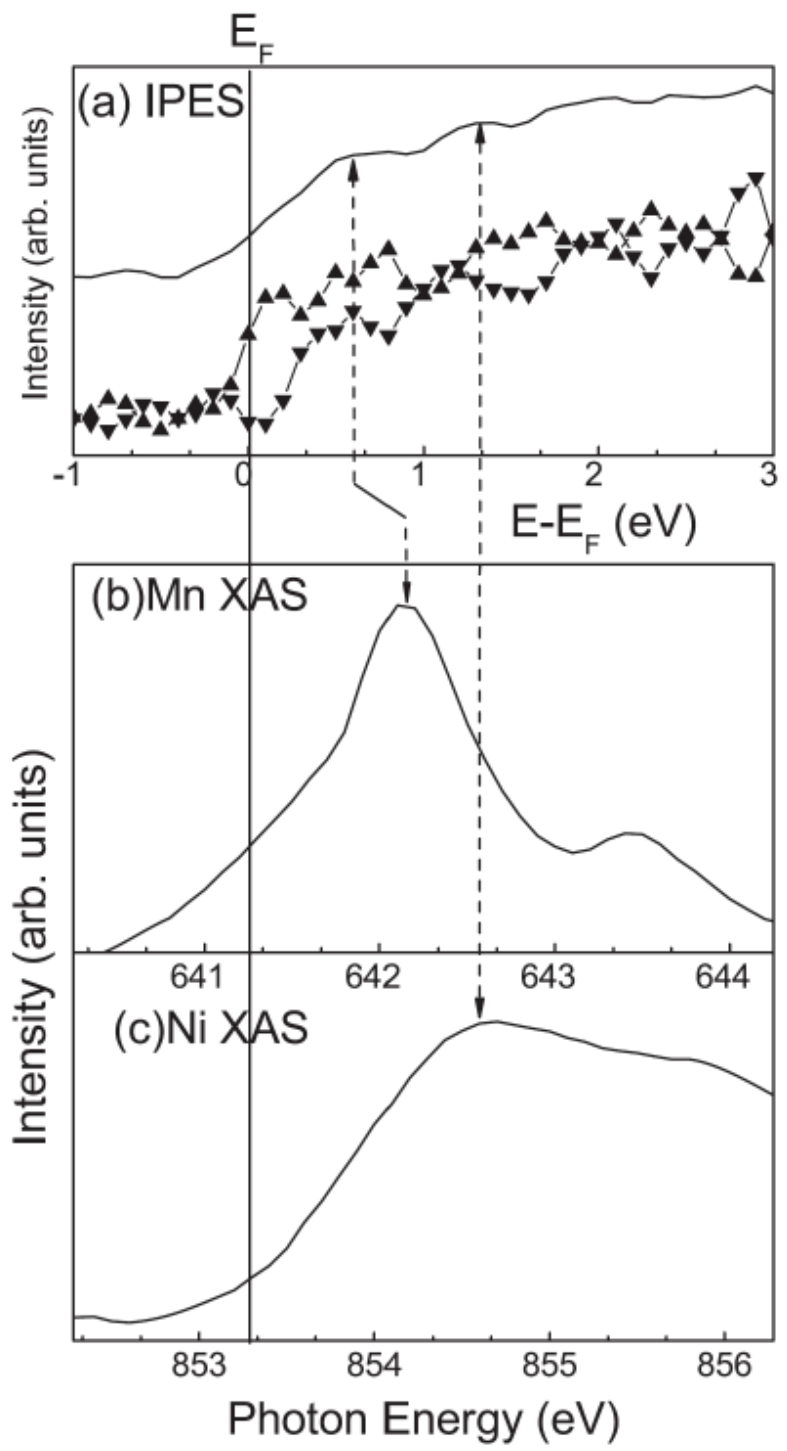

Figure 30. The comparison of the SPIPES, MCD and XAS spectra for NiMnSb(100). The spin polarized inverse photoemission (SPIPES) ( $\boldsymbol{\Delta}$ : spin up, $\boldsymbol{\nabla}:$ spin down) and integrated (continuous line) data for the stoichiometric $\mathrm{NiMnSb}(100)$ clean surface at 300 $\mathrm{K}$ (a) are compared x-ray absorption results of Mn 2p (b) and Ni 2p (c) core-hole spectra. Adapted from [33].

tial state (and could include the expected $\mathrm{d}^{4}$ contributions) [113] at all temperatures from 45 to $250 \mathrm{~K}$. The $\mathrm{Ni} \mathrm{d}^{9}$ initial state contribution to the joint density of unoccupied states measured in IPES is further away from the Fermi level, at approximately $1.3 \mathrm{eV}$.

It is now clear that NiMnSb(100) does have surface states. Theoretically, these surface states are spin minority, and cross the Fermi level away from $\bar{\Gamma}[9,38,42-46,105]$. Indeed there is now experimental confirmation of at least some surface states at the surface of $\mathrm{NiMnSb}$ (100) [105]. 


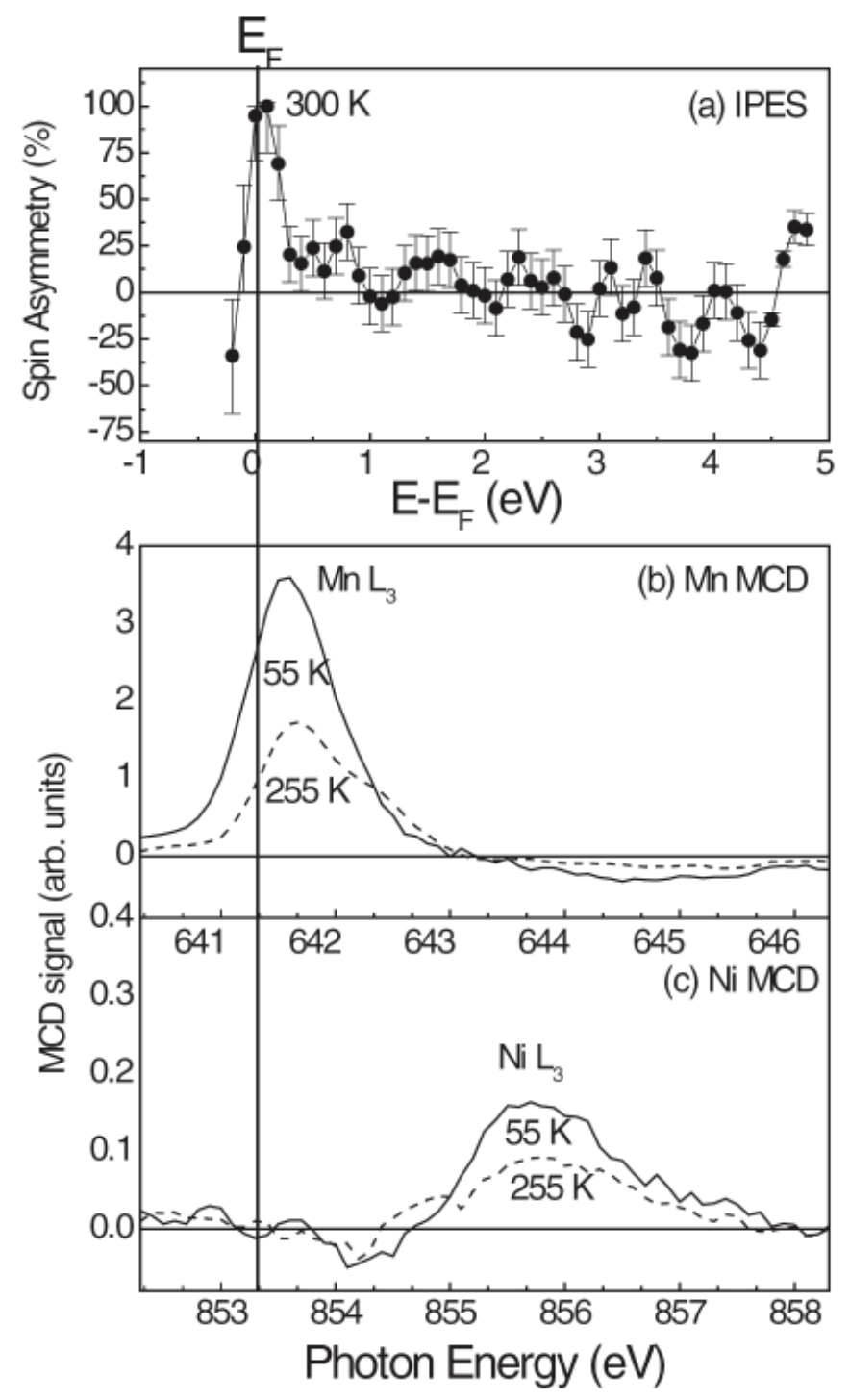

Figure 31. The spin asymmetry obtained in SPIPES at $300 \mathrm{~K}$ is compared with MCD signal across both the $\mathrm{L}_{2}$ and $\mathrm{L}_{3}$ edges of $\mathrm{Mn}(\mathrm{b})$ and $\mathrm{Ni}(\mathrm{c})$ at $55 \mathrm{~K}$ and $255 \mathrm{~K}$. Adapted from [33].

\section{Evidence for temperature dependent magnetic moment ordering in NiMnSb films}

The polarization of the clean $\mathrm{NiMnSb}(100)$ surface is determined by the difference of spin up and down in spin polarized IPES (figure 26). These results are compared with the MCD signal, which is obtained as the difference between XAS signals with opposite magnetization directions, as indicated in figure 31 . The large polarization asymmetry and the strong contribution from the $\mathrm{Mn}$ spin and orbital moments, at remanence, are apparent in the region close to the Fermi level (nearly $100 \%$ above background for $k_{\|}=0$ ).

Since polarization at the Brillouin zone center $\left(k_{\|}=0\right)$ does correspond to long range magnetic order, it is not clear whether the polarization may, in fact, be easily related to 
the magnetic moment for any given system. Just above the Fermi level, spin polarized inverse photoemission measurements provide a polarization close to $100 \%$ at $300 \mathrm{~K}$, in Brillouin zone center $\left(k_{\|}=0\right)$ (figure 31(a)). As the temperature is decreased below $80 \mathrm{~K}$, the MCD exhibits a huge increase in relative spin and orbital moment, as shown in figure 31(b) and (c). Yet in XAS, the apparent density of states near the Fermi level (threshold) does not change significantly for either $\mathrm{Mn}$ or $\mathrm{Ni}$. Therefore, the fact the polarization of $\mathrm{NiMnSb}(100)$ is close to $100 \%$ at an elevated finite temperature ( $300 \mathrm{~K}$ here) at the Brillouin zone centre $\left(k_{\|}=0\right)$ is not a good indicator of the maximum possible remanent band moment or, indeed, of the total "integrated" band polarization in the NiMnSb system, as was noted previously.

Because magnetic circular dichroism is sensitive to band structure polarization (dependent upon symmetry and wavevector), to the remanent unoccupied band polarization, to the magnetocrystalline anisotropy (the crystal field) $[114,115]$, to the excitation cross sections (particularly in non-transmission absorption geometries) and to an often undetermined surface contribution, MCD does not accurately probe the saturated local moment of the bulk material. More accurate measurement of the local bulk sample moment can be obtained from neutron scattering (although this is a less sensitive measure of the global moment that includes local fluctuations). The Fourier transform of the magnetic scattering factor, from neutron scattering, again demonstrates that most of the moment is localized on the Mn sites [59]. The total moment from high field magnetometry (at $10 \mathrm{~K}$ ) is 4.025 $\mu_{\mathrm{B}}$ per formulae unit, consistent with half-metallic character (we attribute the possible excess moment to altered stoichiometry and/or polarization of the inner shells). From polarized neutron scattering we find the local moment on the Mn at $15 \mathrm{~K}, 3.79 \pm 0.02 \mu_{\mathrm{B}}$, falls to $3.55 \pm 0.02 \mu_{\mathrm{B}}$ at $260 \mathrm{~K}$, while the local moment on the Ni sites change little, with values varying around $0.18 \pm 0.02 \mu_{\mathrm{B}}$, so that the total unit cell moment is some $3.98 \pm 0.03$ $\mu_{\mathrm{B}} /$ atom [48], as noted previously. The fact that the local moments changes, even under high field conditions, suggests that there is a change in the moment coupling between $\mathrm{Mn}$ and $\mathrm{Ni}$ at a temperature of about $80 \mathrm{~K}$, as is now also suggested by theory [41]. At higher temperatures, above 80 to $100 \mathrm{~K}$, the saturation moment is lower than that expected for a half-metallic ferromagnet.

The temperature dependence of the local magnetic moments of $\mathrm{Mn}$ and $\mathrm{Ni}$ were obtained by integrating the MCD signals across both the $\mathrm{L}_{3}$ and $\mathrm{L}_{2}$ edge, or only across the $\mathrm{L}_{3}$ edge, at each temperature. As shown in figure 32, the MCD data provide clear evidence of an increased moment ordering in the case of Mn, with its persistent strong local moment. The moment increase is also observed below $80 \mathrm{~K}$ in the case of $\mathrm{Ni}$, and is accentuated if the MCD signals are integrated across both $\mathrm{L}_{3}+\mathrm{L}_{2}$ edges.

The observed increase in the local moment, on the $\mathrm{Mn}$ in neutron scattering, and on the $\mathrm{Mn}$ and $\mathrm{Ni}$ atoms in NiMnSb below 80-100 K in MCD (figures 32 and 33(a) and (b)) are consistent with the increase in the change in $(\Delta \rho / \rho)$ magnetoresistance below $80 \mathrm{~K}$ (shown in figures 14 and 33(d)), and the increase in the $\langle 200\rangle$ neutron magnetic scattering factor, as indicated in figure 15. As noted previously, the resistance of NiMnSb with temperature fits a $T^{1.65}$ power law above $80 \mathrm{~K}$ (close to $T^{3 / 2}$ ), but follows a $T^{2}$ power law below $80 \mathrm{~K}$, as shown in figures 12 and 33(c). The temperature dependence of the resistivity is therefore also consistent with a crossover in magnetic ordering at 80-100 K, though the material remains ferromagnetic. Fitting the $\mathrm{MCD} \mathrm{L}_{3}$ edge data in figure 32 to an Arrhenius expression (consistent with moment fluctuations), indicates that the critical temperature for the classical ferromagnetic to half-metallic ferromagnetic (moment ordering) phase transition is between 80 and $100 \mathrm{~K}$.

The changes in the magnetic structure factor(s), shown in figure 15, suggest a loss in the strong local moment ordering and/or alignment between $\mathrm{Ni}$ and $\mathrm{Mn}$. The change in structure factor suggests a subtle rearrangement of the magnetization density between $\mathrm{Ni}$ 


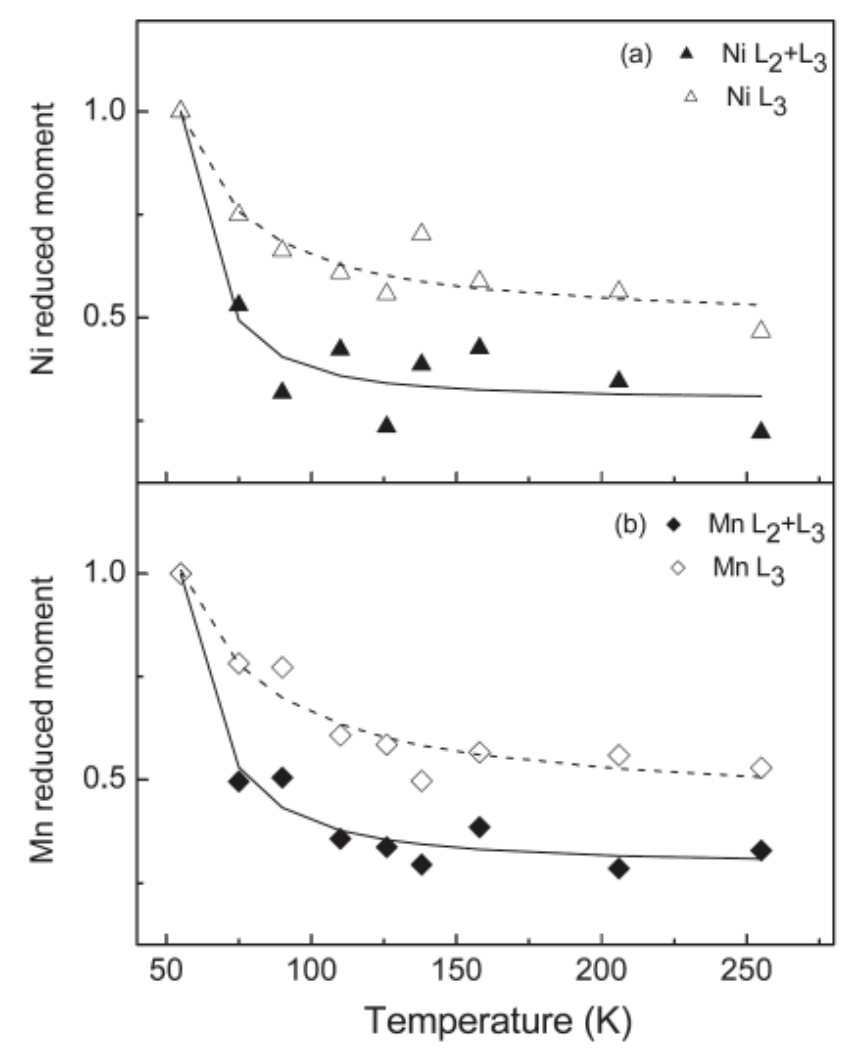

Figure 32. The relative Ni (a) and Mn (b) local moment derived from MCD. The relative moment, obtained by normalization with the value at lowest temperature, contains both spin and orbital contributions from the $\mathrm{L}_{3}$ edge (open symbols) and the sum of both the $\mathrm{L}_{3}$ and $\mathrm{L}_{2}$ edges (filled symbols). Adapted from [33].

and Mn. The transfer of only a small amount of electron density, say just a few hundredths of an electron to the down spin subband or from $\mathrm{Mn}$ to $\mathrm{Ni}$, may not result in much change in either the total moment or the Mn saturation moment (say by about $0.05 \mu_{\mathrm{B}}$ ), but could substantially change the net band polarization near the Fermi level [41]. This effect tends to appear as though there is a failure to completely saturate the magnetization in the MCD measurements, taken at remanence, with increasing temperature. The large change in the MCD signal derived moment compared to the smaller change in neutron scattering moment suggests a large change in the magnetocrystalline anisotropy and band polarization with temperature, although the extremely low effective Debye temperature in the surface region [93] presents a complication to the interpretation of the MCD data that cannot be ignored. Nearest neighbor moment fluctuations [41], resulting in orbital reorientations, would reconcile the spin polarized inverse photoemission and the MCD with the neutron scattering as in the spin polarized inverse photoemission nearest neighbor interactions would only be observed away from the Brillouin zone centre. The proof of temperature dependent orbital hybridization rests on the polarization dependent $\mathrm{x}$-ray absorption spectroscopy described previously.

For the NiMnSb surfaces with a $\mathrm{C}_{2 \mathrm{v}}$ symmetry, the intensity of the $\mathrm{s}$ and p polarized absorption signals can be written as [116]:

$$
\begin{aligned}
& I_{\mathrm{S}}=I_{2,2}+\frac{1}{2} I_{2,1}+\frac{1}{6} I_{2,0} \\
& I_{\mathrm{P}}=I_{2,1}+\frac{2}{3} I_{2,0},
\end{aligned}
$$




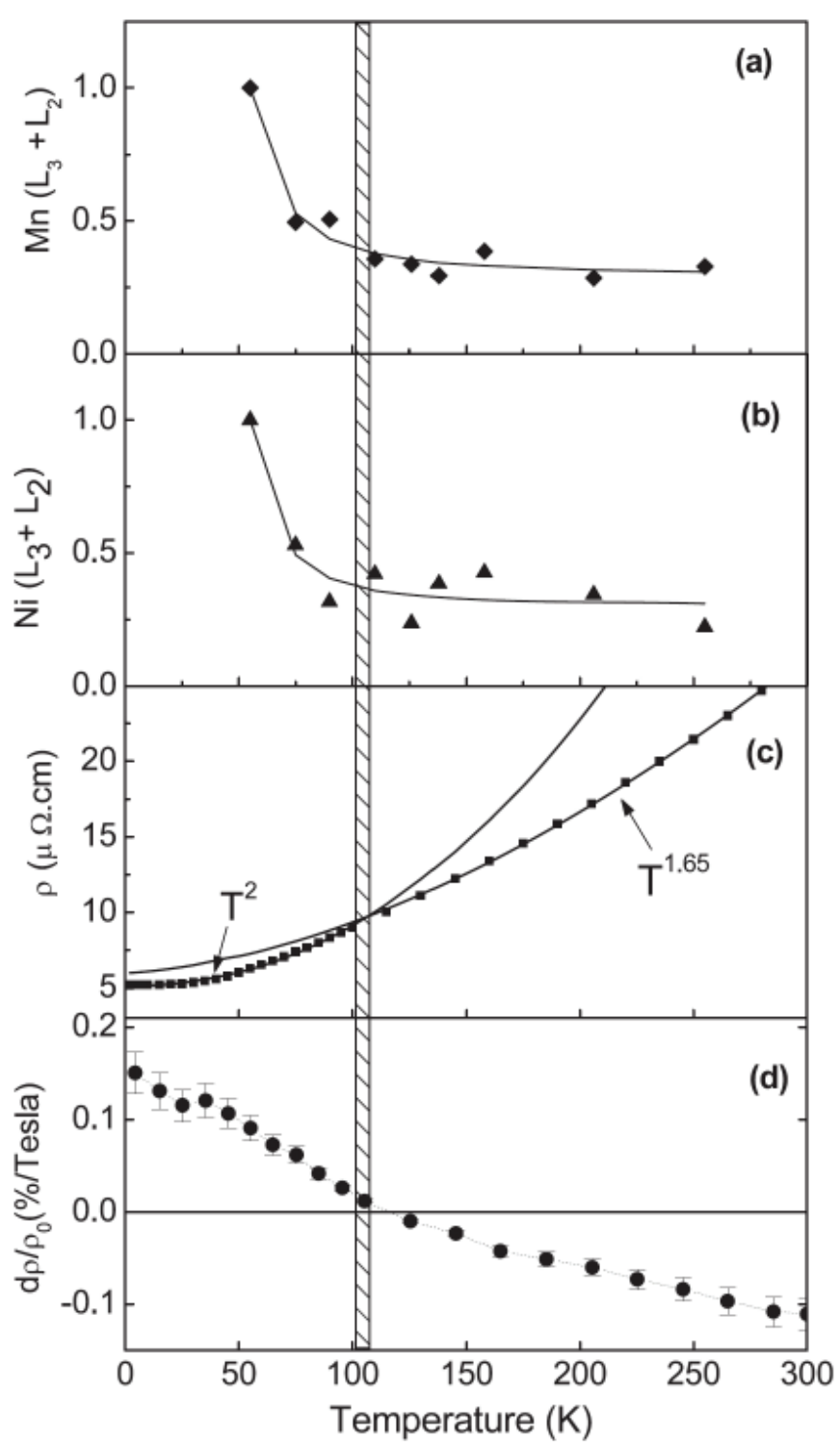

Figure 33. The (a) Mn and (b) Ni relative magnetic moments derived from MCD from the sum of $\mathrm{L}_{3}$ and $\mathrm{L}_{2}$ edges, fitted to an Arrhenius expression and plotted as a function of temperature. (c) The resistance and (d) the longitudinal magnetoresistance slope $\left(\mathrm{d} \rho / \rho_{0}\right)$ taken between 2 and $4 \mathrm{~T}$ as a function of temperature. Note that the changes in the moment observed in MCD compare well with the onset of changes in the magnetoresistance and resistance in NiMnSb. The $T^{2}$ and $T^{1.65}$ curves fitted to the resistance data are as described in the text. Adapted from [33].

where $I_{l, m l}$ is the intensity of the final state transitions of quantum numbers $\left(l, m_{l}\right)$. The corresponding $\mathrm{d}$ orbitals are: $\mathrm{d}_{x^{2}-y^{2}}$ and $\mathrm{d}_{x y}$ for $m=2, \mathrm{~d}_{X Z}$ and $\mathrm{d}_{Y Z}$ for $m=1$, and $\mathrm{d}_{Z^{2}}$ for $m$ $=0$. It is therefore possible to distinguish the contribution of each $\mathrm{d}$ orbital in the total absorption signal. Based on this we can conclude on the hybridization of the unoccupied $d$ orbitals in the conduction band, which correspond to the electron states that are accessed in transport measurements. 


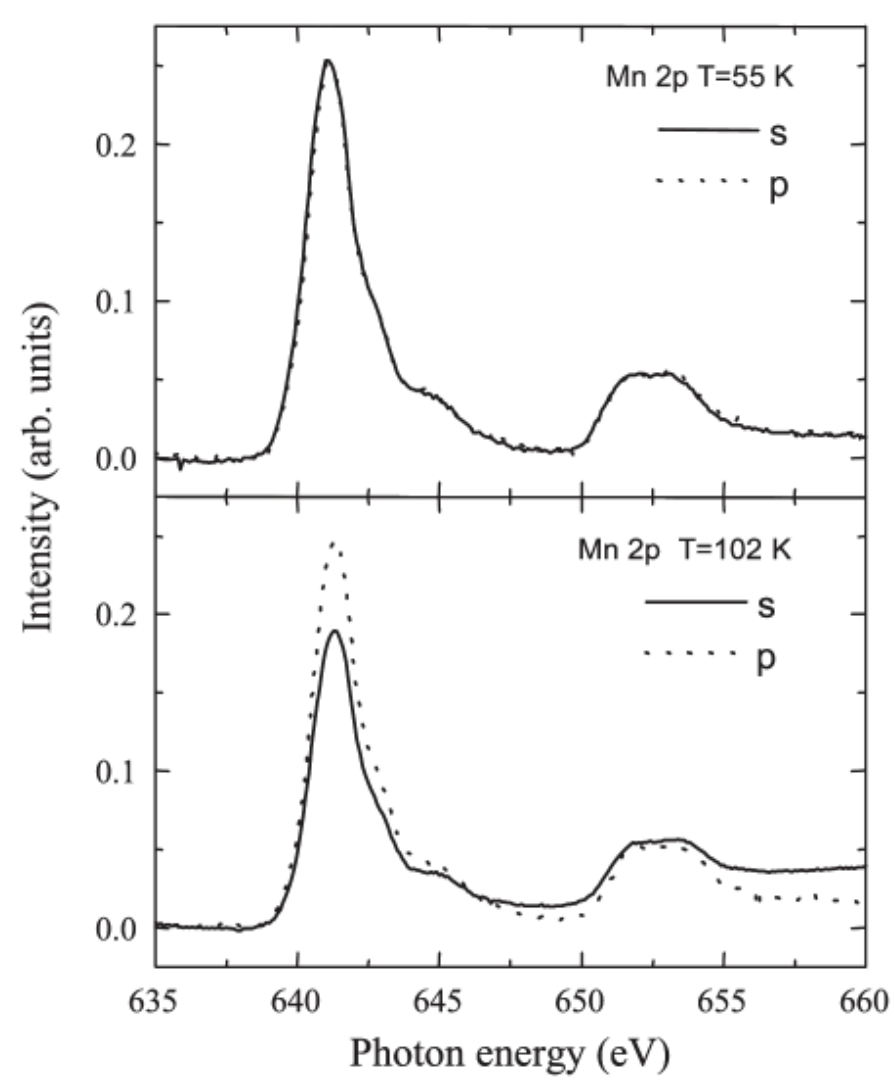

Figure 34. Polarized spectrum $E \| z(\mathrm{p}=$ dotted line $)$ and $E \perp z(\mathrm{~s}=$ continuous line $)$ at 55 $\mathrm{K}$ (upper panel) and $102 \mathrm{~K}$ (lower panel) for the $\mathrm{Mn} \mathrm{L}_{2,3}$ edges measured by XAS in the total electron yield mode.

Figures 34 and 35 show the absorption spectra at the $2 \mathrm{p}$ core levels of the $\mathrm{Mn}$ and $\mathrm{Ni}$ atoms below $(55 \mathrm{~K})$ and above $(102 \mathrm{~K})$ the metamagnetic transition temperature at $T=80$ $\mathrm{K}$, measured in s- and $\mathrm{p}$ polarized incident light. We can clearly see a change in the normalized intensities for the two polarizations as a function of temperature. In the case of the $\mathrm{Mn}$ atom at $55 \mathrm{~K}$, the $\mathrm{s}$ and p polarized spectra are almost the same, while at $102 \mathrm{~K}$ the p polarized intensity is enhanced. The reverse is true for the $\mathrm{Ni}$ atom-the s polarized spectrum is bigger than p polarized spectrum at $55 \mathrm{~K}$ while at $102 \mathrm{~K}$ the $\mathrm{Ni}$ absorption spectra show almost same intensity.

According to equations (21), we expect an enhanced p polarized spectra compared to the s polarized one when the absorption on the $m_{l}= \pm 2\left(I_{2,2}\right)$ states is reduced. When the two linearly polarized spectra are equal, then the $I_{2,2}$ signal must have an important (medium) contribution in order to compensate the $1 / 2 I_{2,1}+1 / 2 I_{2,0}$ signal. Finally, for the case when the s polarized spectrum is more intense than the $\mathrm{p}$ polarized one, then the $I_{2,2}$ signal must dominate.

This interpretation can be translated into a reduced $\mathrm{d}_{x^{2}-y^{2}}$ and $\mathrm{d}_{x y}$ orbital contribution to the hybridization between $\mathrm{Mn}$ and $\mathrm{Ni}$, as the temperature increases. In fact, in case of the $\mathrm{Mn}$ atom at low temperatures, we start from a medium contribution $\left(I_{\mathrm{P}}=I_{\mathrm{S}}\right)$ of the $I_{2,2}$ signal to the total intensity and we arrive to a low contribution $\left(I_{\mathrm{P}}>I_{\mathrm{S}}\right)$, at higher temperatures. For the Ni atom, we begin with a strong contribution $\left(I_{\mathrm{P}}<I_{\mathrm{S}}\right)$ of the $I_{2,2}$ signal to the total intensity at low temperatures and end up with a medium contribution $\left(I_{\mathrm{P}}=I_{\mathrm{S}}\right)$ at higher temperatures. 


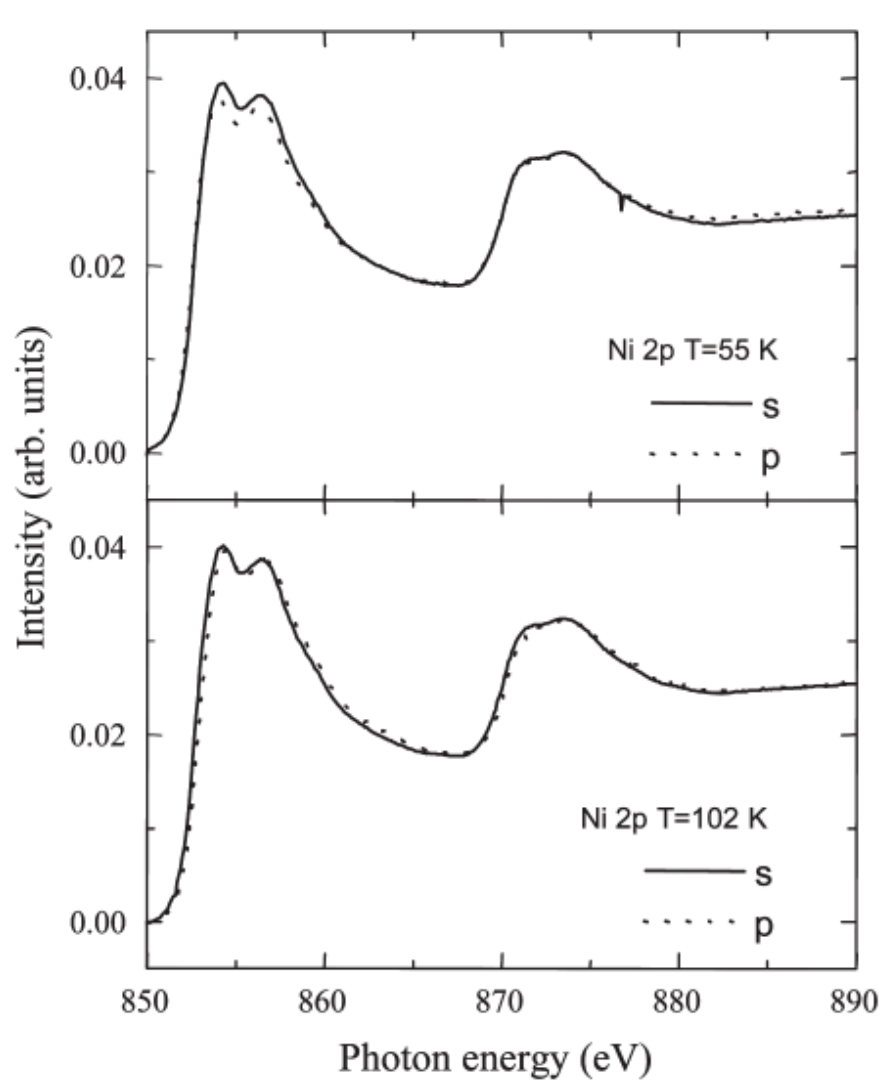

Figure 35. Polarized spectrum $E \| z(\mathrm{p}=$ dotted line $)$ and $E \perp z$ (s = continuous line) at 55 $\mathrm{K}$ (upper panel) and $102 \mathrm{~K}$ (lower panel) for the $\mathrm{Ni}_{2,3}$ edges measured by XAS in the total electron yield mode.

Taking into account the geometry of the Mn and Ni sites in the NiMnSb crystal lattice, the best orbital overlap is achieved between the $\mathrm{d}_{x^{2}-y^{2}}$ and $\mathrm{d} x y$ orbitals of $\mathrm{Mn}$ and the $\mathrm{d}_{X Z, Y Z}$ or $\mathrm{d}_{Z 2}$ orbital of $\mathrm{Ni}$. On the contrary, the hybridizations between the $\mathrm{d}_{X Z, Y Z}$ and $\mathrm{d}_{Z 2}$ orbitals of the two atoms are not very strong. Since at $55 \mathrm{~K}$ the $I_{2,2}$ intensity of Ni is strong, and the one of $\mathrm{Mn}$ is medium, while at $102 \mathrm{~K}$ the $I_{2,2}$ intensity of Ni is medium and the one of $\mathrm{Mn}$ is weak, we must have an enhanced hybridization between the $\mathrm{d}_{x^{2}-y^{2}}$ with $\mathrm{d}_{X Z, Y Z}$ or between the $\mathrm{d}_{x y}$ with $\mathrm{d}_{Z 2}$ orbitals at low temperatures compared to higher temperatures. Therefore, we conclude that the hybridization or the alignment between the $\mathrm{Mn}$ and Ni orbital reduces as the temperature increases, as is now suggested from theory [41]. In other words, an orbital reorientation takes place as a function of temperature, switching from a strong hybridization at $55 \mathrm{~K}$ to a weak one at $102 \mathrm{~K}$. This reorientation is the result of magnon and phonon effects at finite temperature [36-38, 41] and accounts for the loss of the very high polarization values above $80 \mathrm{~K}$.

\section{Is the epitaxial NiMnSb film a true half-metal?}

In $\mathrm{NiMnSb}$, the density of states close to the gap is dominated by $\mathrm{d}$ states: in the valence band by bonding hybrids with large Ni admixture and, in the conduction band, by the antibonding hybrids with large Mn admixture. Thus the gap originates from the strong hybridization between the $d$ states of the higher valence and the lower valence transition metal atoms. While the $\mathrm{Sb}$ atoms (having only s and $\mathrm{p}$ states) are not responsible for the 
existence of the minority gap, their bands strongly hybridize with the transition metals $\mathrm{d}$ states such that the charges in the $s, p$ bands are delocalized. Changing the sp element or changing the orbital hybridization may result in a reduction in polarization [41]. The latter can occur not simply due to defects but also due to spin fluctuations [41] so that the spin minority gap has a maximum value only at $T=0 \mathrm{~K}[36-38,41]$. At finite temperatures, spin disorder increases the spin down density from zero to a non-zero value in the middle of the spin down gap, way above the highest energy level occupied at zero temperature. As a result, the resistance of the spin down channel changes from infinity to a finite value. But even at zero $\mathrm{K}$, the non-quasiparticle density of states $[38,61,62]$ should have a small effect and reduce the polarization below $100 \%$.

The multisublattice character of most half-metallic ferromagnets, which is most pronounced in zero-magnetic-field conditions, yields transverse optical phonon modes [3638 ] which couple to thermally excited magnons. It may be that longitudinal modes may prove to be even more important [41]. As we can see in figure 25, nearly dispersionless transverse and longitudinal optical modes are observed around $28 \mathrm{meV}$. Magnon coupling to these phonon modes should result in a dramatic loss in $\mathrm{Ni}$ and $\mathrm{Mn}$ magnetic moments and therefore, in overall polarization of the NiMnSb. This corresponds to tilting between neighboring $\mathrm{Ni}$ and $\mathrm{Mn}$ atoms or to orbital reorientation occurring around $80 \mathrm{~K}$, as shown in section 7. Other models of the temperature dependence of the polarization, due to spin waves have been proposed [41, 44]. It is difficult to envisage how magnons can be eliminated from potential half-metallic systems having more than one sublattice, in order to ensure the true half-metallic character.

At temperatures that are above magnon dominated depolarization, the thermally activated spin mixing mechanism is enhanced by crystal imperfections and by surface and interface effects. Figure 22 showed that a strong chemical potential for surface segregation is present in NiMnSb, indicating that the equilibrium surface has not the same stoichiometry as the bulk thin film [48].

As noted much earlier, the value of $100 \%$ spin polarization measured at $300 \mathrm{~K}$ at the center of the Brillouin zone, seen in figure 26, does not provide the evidence of half-metallic behavior. Polarization at $k_{\|}=0$ corresponds to long range magnetic order but is only loosely related to the magnetic moment. The data shown in figure 30 suggest a very high spin polarization near the Fermi level at $300 \mathrm{~K}$, but as temperature is increased above 80 $\mathrm{K}$, the MCD indicates a decrease in the relative band structure polarization ( $k$ integrated). The finite temperature effects, leading to the population of spin minority states near the Fermi level, would be most significant at wavevectors away from $k_{\|}=0$. Similarly, spin minority surface states would also be more evident at wavevectors away from $k_{\|}=0$ [9, $38,42-46,105]$. Thus neither might be observed by spin polarized inverse photoemission (or spin polarized photoemission) at normal incidence.

Whatever the origin of the depolarization, and the influence of spin waves, half-metallic ferromagnetism should be treated as an idealized limit, realistic only in perfect crystals at zero temperature; if correlation effect are considered, then the non-quasiparticle density of states forbids perfect polarization even in the ground state. Due to finite temperature and surface effects present at the surfaces of $\mathrm{NiMnSb}$ films, the classic ferromagnets like $\mathrm{Co}, \mathrm{Fe}$ or $\mathrm{Ni}$ with less than $100 \%$ spin polarization may be more reliable for spin injection than the half-metallic systems, especially under ambient or elevated temperatures. It appears that nature abhors halfmetallicity [117].

\section{Summary}

High quality epitaxial NiMnSb thin films have been deposited by facing targets sputtering, using $\mathrm{MgO}$ monocrystalline substrates with Mo buffer layers. The epitaxial growth 
relation has been found to be (100)NiMnSb \| (100)Mo $\|$ (110)MgO. Optimum deposition conditions have been determined to achieve highly ordered, continuous films with a low rms roughness. The magnetic and transport properties of the films are comparable to those of bulk single crystals, with the residual resistivity being much lower than what has been reported so far in the literature.

It has been clearly shown by us and others that the surface of $\mathrm{NiMnSb}(100)$ is very fragile and surface structure and composition can be easily altered by thermal treatments. The standard sputter and annealing treatments, used to clean most surfaces, lead to $\mathrm{Mn}$ segregation or some other surface termination, rarely the stoichiometric surface. For the well-prepared, nearly stoichiometric ordered alloy, the surface appears to terminate in $\mathrm{MnSb}$ in agreement with theoretical expectations. Vibrational amplitude of the atoms in the surface and selvedge region were evaluated by extracting the effective Debye temperature using LEED and XPS. Comparing the bulk Debye temperature, obtained by neutron scattering, with the effective Debye temperature of the surface, we find that surface Debye temperature is almost $45 \%$ of the bulk value. This drop between the surface and the bulk Debye temperatures can be understood if we take into account that the surface and selvedge regions retain a different composition than the bulk.

The use of different surface preparation methods results in modifications in the surface electronic structure, as theoretically predicted $[49,50,118]$. The general trend is that surface disorder destroys the spin asymmetry near the Fermi level and, therefore, that the assumed half-metals become normal ferromagnets. The maximum spin asymmetry obtained in $k_{\|}=0$ for a stoichiometric NiMnSb surface was approximately $100 \%$ at room temperature. As soon as the Mn segregation is induced by the difference in enthalpy between the surface and the bulk regions, the spin asymmetry at the Fermi level drops to a negative value, which is more reminiscent of $\mathrm{MnSb}$ binary alloy or an antiferromagnetically aligned $\mathrm{Mn}$ rich surface layer.

The electronic bands closest to the Fermi level are assigned almost entirely to the $\mathrm{Mn}$ $3 \mathrm{~d}$ bands. The valence band of NiMnSb contains a high degree of overlapping between Ni 3d and Mn 3d bands [8, 41, 44]. The first peak in the conduction band of NiMnSb has pure Mn character, which accounts for the high polarization measured at $k_{\|}=0$.

While it has been suggested that NiMnSb is a half-metallic system [1, 24, 112], it is certainly clear, from the data presented here, that NiMnSb is not a half-metallic ferromagnet above the transition at about $80-100 \mathrm{~K}$, although still well below the ferromagnetic Curie temperature of $\sim 730 \mathrm{~K}$. Amongst the other potential half-metallic systems, the manganese perovskites have exhibited a number of phase transitions well below the ferromagnetic $T_{\mathrm{c}}[94,97]$. The presence of these phase transitions (affecting the moment in the case of $\mathrm{NiMnSb}$ ) makes clear that finite temperature calculations and measurements that probe the polarization of Fermi level crossings are essential to establish half-metallic ferromagnetism at any appreciable temperature, particularly for the surface. Neither $100 \%$ polarization at one $k$ point nor $T^{3 / 2}$ temperature dependent resistance (sometimes taken as indicative of a half-metallic ferromagnetic $[24,119]$ ) are good indicators of half-metallic behavior.

\section{Acknowledgments}

This work was supported by Nebraska NSF MRSEC (DMR-0213808), the Nebraska Research Initiative and the Office of Naval Research (Grant No N00014-06-1-0616). The authors would like to thank S. Andrieu and A. Traverse for their help with the EXAFS measurements, S. Stadler for his assistance with the MCD measurements and C. Hordequin, J. Pierre, and R. Currat for performing the neutron scattering experiments. 


\section{References}

[1] de Groot R A and Mueller F M 1983 Phys. Rev. Lett. 502024

[2] de Groot R A, Mueller F M, van Engen P G, and Buschow K H J 1984 J. Appl. Phys. 552151

[3] Kübler J 1984 Physica B 127257

[4] Fang C M, de Wijs G A, and deGroot R A 2002 J. Appl. Phys. 918340

[5] Hanssen K E H M and Mijnarends P E 1986 Phys. Rev. B 345009

[6] Galanakis I, Ostanin S, Alouani M, Dreysse H, and Wills J M 2000 Phys. Rev. B 614093

[7] Kang J-S, Park J-G, Olson C G, Youn S J, and Min B I 1995 J. Phys.: Condens. Matter 7789

[8] Kang J-S, Hong S H, Jung S W, Lee Y P, Park J-G, Olson C G, Youn S J, and Min B I 1993 Solid State Commun. 88635

[9] Galanakis I 2002 J. Phys.: Condens. Matter 146329

[10] Galanakis I, Dederichs P H, and Papanikolaou N 2002 Phys. Rev. B 66174429

[11] Galanakis I, Dederichs P H, and Papanikolaou N 2002 Phys. Rev. B 66134428

[12] Liu B G 2003 Phys. Rev. B 67172411

[13] Xie W H, Xu Y Q, Liu B G, and Pettifor D G 2003 Phys. Rev. Lett. 91037204

[14] Galanakis I 2002 Phys. Rev. B 66012406

[15] Pickett W E and Singh D J 1996 Phys. Rev. B 531146

[16] Singh D J and Pickett W E 1998 Phys. Rev. B 5788

[17] Kobayashi K-I, Kimura T, Sawada H, Terakura K, and Tokura Y 1998 Nature 395677

[18] Schwarz K 1986 J. Phys. F: Met. Phys. 16 L211

[19] van Lueken H and de Groot R A 1995 Phys. Rev. B 517176

[20] Korotin M A, Anisimov V I, Khomskii D I, and Sawatzky G A 1998 Phys. Rev. Lett. 804305

[21] Lewis S P, Allen P B, and Sasaki T 1997 Phys. Rev. B 5510253

[22] de Groot R A and Buschow K H J 1986 J. Magn. Magn. Mater. 54-57 1377

[23] Penicaud M, Silberchoit B, Sommers C B, and Kubler J 1992 J. Magn. Magn. Mater. 103212

[24] Irkhin V Yu and Katsnelson M I 1994 Phys. Usp. 37659

[25] Mazin I I 2000 Appl. Phys. Lett. 773000

[26] Bona L, Meier F, Taborelli M, Bucher E, and Schmidt P H 1985 Solid State Commun. 56391

[27] Soulen R J et al. 1998 Science 28285

[28] Miyoshi Y, Bugoslavsky Y, Syed M H, Robinson T, Cohen L F, Singh L J, Barber Z H, Grigorescu C E A, Gardelis S, Giapintzakis J, and Van Roy W 2006 Appl. Phys. Lett. 88142512

[29] Sicot M, Turban P, Andrieu S, Tagliaferi A, de Nadai C, Brookes N B, Bertran F, and Fortuna F 2006 J. Magn. Magn. Mater. 30354

[30] Wolfgang G 1991 PhD Thesis Freie University of Berlin, Germany

[31] Kolev H, Rangelov G, Braun J, and Donath M 2005 Phys. Rev. B 72104415

[32] Ristoiu D, Nozières J P, Borca C N, Komesu T, Jeong H-K, and Dowben P A 2000 Europhys. Lett. 49624

[33] Borca C N, Takashi K, Jeong H-K, Dowben P A, Ristoiu D, Hordequin Ch, Nozières J P, Pierre J, Shane S, and Idzerda Y U 2001 Phys. Rev. B 64052409

[34] Ristoiu D 2000 PhD Thesis Université Joseph Fourier, Grenoble I, France

[35] Skomski R and Dowben P A 2002 Europhys. Lett. 58544

[36] Dowben P A and Skomski R 2003 J. Appl. Phys. 937948

[37] Dowben P A and Skomski R 2004 J. Appl. Phys. 957453

[38] Dowben P A and Jenkins S J 2005 Frontiers in Magnetic Materials ed A Narlikar (Berlin: Springer) pp 295-325

[39] Itoh H, Ohsawa T, and Inoue J 2000 Phys. Rev. Lett. 842501

[40] MacDonald A H, Jungwirth T, and Kasner M 1998 Phys. Rev. Lett. 81705

[41] Lezaic M, Mavropoulos P, Enkovaara J, Bihlmayer G, and Blugel S 2006 Phys. Rev. Lett. 97026404 
[42] de Wijs G A and de Groot R A 2001 Phys. Rev. B 64020402

[43] Jenkins S J and King D A 2001 Surf. Sci. 494 L793

[44] Lezaic M, Galanakis I, Bihlmayer G, and Blugel S 2005 J. Phys.: Condens. Matter 173121

[45] Lezaic M, Mavropoulos P, Bihlmayer G, and Blugel S 2006 J. Phys. D: Appl. Phys. 39797

[46] Galanakis I 2005 J. Magn. Magn. Mater. 288411

[47] Tanaka C T, Nowak J, and Moodera J S 1999 J. Appl. Phys. 866239

[48] Ristoiu D, Nozières J P, Borca C N, Borca B, and Dowben P A 2000 Appl. Phys. Lett. 762349

[49] Orgassa D, Fujiwara H, Schulthess T C, and Butler W H 1999 Phys. Rev. B 6013237

[50] Orgassa D, Fujiwara H, Schulthess T C, and Butler W H 2000 J. Appl. Phys. 875870

[51] Hordequin C, Pierre J, and Currat R 1996 J. Magn. Magn. Mater. 16275

[52] Hordequin C, Ristoiu D, Ranno L, and Pierre J 2000 Eur. Phys. J. B 16287

[53] Ristoiu D, Nozières J P, and Ranno L 2000 J. Magn. Magn. Mater. 21997

[54] Hordequin C, Nozières J P, and Pierre J 1998 J. Magn. Magn. Mater. 183225

[55] Lewis B and Anderson J C 1978 Nucleation and Growth of Thin Films (New York: Academic)

[56] Thornton J A 1982 Coating Deposition by Sputtering. Deposition Technologies for Films and Coatings (Park Ridge, NJ: Noyes Publications)

[57] Michalowizc A 1990 Thèse d'état Univ. Paris Val de Marne

[58] McKale A G 1988 J. Am. Chem. Soc. 1103763

[59] Hordequin C, Lelievre-Berna E, and Pierre J 1997 Physica B 234602

[60] Skomski R, Zhou J, Dowben P A, and Sellmyer D J 2005 J. Appl. Phys. 97 10C305

[61] Chioncel L, Katsnelson M I, de Groot R A, and Lichtenstein A I 2003 Phys. Rev. B 68144425

[62] Irkhin V Y and Katsnelson M I 1985 J. Phys. C: Solid State Phys. 184173

[63] Otto M J, van Woerden R A M, van der Valk J P, Wijngaard J, van Bruggen C F, and Haas C 1989 J. Phys.: Condens. Matter 12351

[64] Moodera J S and Mootoo D M 1994 J. Appl. Phys. 766101

[65] Dowben P A and Miller A (ed) 1990 Core-level Spectroscopy and the Characterization of Surface Segregation, in Surface Segregation Phenomena (Boston, MA: CRC Press)

[66] Choi J, Zhang J, Liou S-H, Dowben P A, and Plummer E W 1999 Phys. Rev. B 5913453

[67] Dulli H, Dowben P A, Liou S-H, and Plummer E W 2000 Phys. Rev. B 62 R14629

[68] Borca C N, Xu B, Komesu T, Jeong H-K, Liu M T, Liou S-H, and Dowben P A 2002 Surf. Sci. Lett. 512 L346

[69] Seah M P, Jones M E, and Anthony M T 1984 Surf. Interface Anal. 6 242; Seah M P 1993 Surf. Interface Anal. 20243

[70] Monnier R 1997 Phil. Mag. B 7567

[71] Foiles S M 1985 Phys. Rev. B 327685

[72] Finnis M W and Sinclair J E 1984 Phil. Mag. A 5045

[73] Bozzolo G, Ferrante J, Noebe R D, Good B, Honecy F S, and Abel P 1999 Comput. Mater. Sci. 15 169

[74] Deng H, Hu W, Shu X, and Zhang B 2004 Appl. Surf. Sci. 221408

[75] Jenkins S J 2004 Phys. Rev. B 70245401

[76] Donath M and Nolting W (ed) 2005 Local Moment Ferromagnets (Berlin: Springer) p 245

[77] Tanuma S, Ichimura S, and Yoshihara K 1996 Appl. Surf. Sci. 10147

[78] NIST Electron Inelastic Mean Free Path Program Version 1.1 (NST std. ref. Database 71); http://www.nist.gov.srd

[79] Jeong H K, Komesu T, Dowben P A, Schultz B D and Palmstrøm C J 2002 Phys. Lett. A 302217

[80] Briggs D and Seah M P (ed) 1990 Practical Surface Analysis-Auger and X-ray Photoelectron Spectroscopy vol 1 (Chichester: Wiley)

[81] Scofield J H 1976 J. Electron Spectrosc. Relat. Phenom. 8129

[82] Penn D R 1976 J. Electron Spectrosc. Relat. Phenom. 929 
[83] Liu S Y and Kung H H 1981 Surf. Sci. 110504

[84] Alling B, Shallcross S, and Abrikosov I A 2006 Phys. Rev. B 73064418

[85] Attema J J, Fang C M, Chioncel L, de Wijs G A, Lichtenstein A I, and de Groot R A 2004 J. Phys.: Condens. Matter 16 S5517

[86] Wang S C and Ehrich G 1988 Surf. Sci. 206457

[87] Roux H, Piquet A, Uzun R, and Drechsler M 1976 Surf. Sci. 5997

[88] Badorf A D and Plummer E W 1990 J. Electron Spectrosc. Relat. Phenom. 54/55 541

[89] Badorf A D and Plummer E W 1991 Phys. Rev. Lett. 662770

[90] Benedeck G and Toennies J P 1992 Phys. Rev. B 4613643

[91] Tung R T, Graham W R, and Melmed A J 1982 Surf. Sci. 115576

[92] King D A and Thomas G 1980 Surf. Sci. 91201

[93] Borca C N, Ristoiu D, Komesu T, Jeong H K, Hordequin C, Pierre J, Nozières J P, and Dowben P A 2000 Appl. Phys. Lett. 7788

[94] Borca C N, Choi J, Adenwalla S, Ducharme S, Dowben P A, Robertson L, Fridkin V M, Palto S P, and Petukhova N 1999 Appl. Phys. Lett. 74347

[95] Zhang J and Plummer E W 1997 Surf. Sci. 39364

[96] Clarke L J 1985 Surface Crystallography—An Introduction to Low Energy Electron Diffraction (New York: Wiley)

[97] Waldfried C, McIlroy D N, Zhang J, Dowben P A, Katrich G A, and Plummer E W 1996 Surf. Sci. 363296

[98] van Hove M A, Weinberg W H, and Chan C-M 1986 Low-Energy Electron Diffraction (Springer Series in Surface Science) vol 6 (Berlin: Springer) p 134

[99] Gopal E S R 1966 Specific Heats at Low Temperatures (New York: Plenum) p 29

[100] Kittel C 1996 Introduction to Solid State Physics (New York: Wiley) p 90

[101] Hordequin C 1997 PhD Thesis Université Joseph Fourier, Grenoble I, France

[102] Yang L and Rahman T S 1991 Phys. Rev. Lett. 672327

[103] Ditlevsen P D, Stoltze P O, and Nørskov J K 1991 Phys. Rev. B 4413002

[104] Borca C N, Komesu T, and Dowben P A 2002 J. Electron. Spectrosc. Relat. Phenom. 122259

[105] Correa J S, Eibl C, Rangelov G, Braun J, and Donath M 2006 Phys. Rev. B 73125316

[106] Coehoorn R, Haas C, and de Groot R A 1985 Phys. Rev. B 311980

[107] Okuda H, Senba S, Sato H, Shimada K, Namatame H, and Taniguchi M 1999 J. Electron. Spectrosc. Relat. Phenom. 101-103 657

[108] Panchula A F, Kaiser C, Kellock A, and Parkin S S P 2003 Appl. Phys. Lett. 831812

[109] Ravidran P, Delin A, James P, Johansson B, Willis J M, Ahuja R, and Eriksson O 1999 Phys. Rev. B 59 15680; Rader O et al. 1998 Phys. Rev. B 57 R689

[110] van der Laan G and Kirkman I W 1992 J. Phys.: Condens. Matter 44189

[111] de Groot F M F, Hu Z W, Lopez M F, Kaindl G, Guillot F, and Tronc M 1994 J. Chem. Phys. 101 6570

[112] Galanakis I, Ostanin S, Alouani M, Dreysse H, and Wills J M 2000 Phys. Rev. B 614093

[113] Thole B T, Cowan R D, Sawatzky G A, Fink J, and Fuggle J C 1985 Phys. Rev. B 316856

[114] Stöhr J and König H 1995 Phys. Rev. Lett. 753748

[115] Dürr H et al. 1996 Phys. Rev. Lett. 763464

[116] Bianconni A, della Longa S, Li C, Pompa M, Congiu-Castellano A, Udron D, Flank A M, and Lagarde P 1991 Phys. Rev. B 4410126

[117] Paraphrase of remarks by Hathaway K 2005 private communication, Office of Naval Research

[118] Sarma D D 1996 J. Electron. Spectrosc. Relat. Phenom. 7837

[119] Imada M, Fujimori A, and Tokura Y 1998 Rev. Mod. Ph 